Mathematical Models and Methods in Applied Sciences

Vol. 21, No. 1 (2011) 29-55

(C) World Scientific Publishing Company

DOI: 10.1142/S0218202511004976

\title{
FERROMAGNETS WITH EDDY CURRENTS AND PINNING EFFECTS: THEIR THERMODYNAMICS AND ANALYSIS
}

\author{
TOMÁŠ ROUBÍČEK \\ Mathematical Institute, Charles University, \\ Sokolovská 83, CZ-186 75 Praha 8, Czech Republic \\ and \\ Institute of Thermomechanics of the ASCR, \\ Dolejškova 5, CZ-182 00 Praha 8, Czech Republic \\ roubicek@karlin.mff.cuni.cz \\ GIUSEPPE TOMASSETTI \\ Dipartimento di Ingegneria Civile, Università di Roma TorVergata, \\ Via Politecnico 1, I-00133 Roma, Italy \\ tomassetti@ing.uniroma2.it \\ Received 12 November 2009 \\ Revised 12 July 2010 \\ Communicated by N. Bellomo and J. Málek \\ Dedicated to K. R. Rajagopal on the Occasion of His 60th Birthday
}

\begin{abstract}
Existence of weak solutions is proved for a system of nonlinear parabolic equations/inequalities describing evolution of magnetization, temperature, magnetic field, and electric field in electrically-conductive unsaturated ferromagnets. The system is derived from a recently-proposed thermodynamically-consistent continuum theory for the ferro/paramagnetic transition. Besides the standard viscous-like damping, dissipation due to eddy currents and domain-wall pinning is considered.
\end{abstract}

Keywords: Micromagnetics; Gilbert equation; heat equation; Joule heat; electrical conduction; eddy currents; pinning; weak solution.

AMS Subject Classification: 35D05, 35K55, 49S05, 78A99, 80A17

\section{Introduction}

Magnetization dynamics in ferromagnetic solids is a very complex phenomenon. Most expositions, especially in the mathematically-oriented literature, cover only particular physical aspects and, with few exceptions, assume that the body is held at constant temperature. In Ref. 21 a continuum model describing the evolution of the 
magnetization has been proposed which differs from previous expositions in two aspects: first, the saturation constraint is suppressed, and a Landau-type term is included in the free energy so as to allow the magnetization intensity to depend on temperature; second, a thermodynamically-consistent coupling mechanism between the Gilbert equation and the heat equation is envisaged. Using this model as starting point, an initial-boundary value problem for the magnetization and temperature fields has been formulated and the existence of weak solutions has been proved in the same paper.

The mathematical analysis carried out in Ref. 21 covers viscous-type dissipation mechanisms. Yet, in ferromagnetic solids other dissipation mechanisms may be important. At very low frequencies, hysteretic response is observed in magnetization/ applied field diagrams (this happens when temperature is stabilized, whereas a more complex response is expected for fast anisothermal processes ${ }^{25}$ ). Crystal imperfections are deemed to be responsible for this phenomenon by raising energy barriers that pin domain walls, with de-pinning taking place only when the external driving force exceeds a threshold. Viscous-like damping cannot account for dissipation mechanisms that persist under slow motions (an analytical argument supporting this statement may be found in Ref. 23). This fact motivates the proposal (see Refs. 4 and 28 ) of adding a dry-friction-like term to the standard viscous-like damping in the Gilbert equation. Analytical results for a model with dry friction may be found in Ref. 26, where, however, temperature is assumed to be constant.

In this paper we illustrate what changes are needed in the mathematical analysis carried out in Ref. 21 to cover dissipation mechanisms associated to domain-wall pinning and eddy currents. Of course, other effects are neglected. In particular, we neglect magnetostriction. We also neglect displacement current and use so-called eddy-current approximation of the full Maxwell system, and we also neglect the electromagnetic field outside the body, cf. Remark 5.2. We also neglect thermoelectrical cross-effects, cf. Remark 5.3.

The paper is organized as follows. In Sec. 2, we formulate an initial-boundaryvalue problem that captures the phenomenology of interest. In Sec. 3, we specify data qualification, give a notion of weak solution, and state our existence theorem. In Sec. 4, we prove existence of a weak solution by a carefully selected succession of approximations. In Sec. 5, we discuss possible extensions of the model.

\section{Problem Formulation}

We consider the following system of partial differential inclusions/equations:

$$
\begin{aligned}
& \frac{1}{g(|\mathrm{~m}|)} \mathrm{m} \times \dot{\mathrm{m}}+\lambda \Delta \mathrm{m}-\varphi_{0}^{\prime}(\mathrm{m})-\theta \varphi_{1}^{\prime}(\mathrm{m})+\mu_{0}\left(\mathrm{~h}+\mathrm{h}_{\mathrm{e}}\right)-\alpha \dot{\mathrm{m}} \in \partial \varrho(\dot{\mathrm{m}}), \\
& c(\theta) \dot{\theta}-\operatorname{div}(\kappa(\theta) \nabla \theta)=\sigma(\theta)|\mathrm{e}|^{2}+\alpha|\dot{\mathrm{m}}|^{2}+\varrho(\dot{\mathrm{m}})+\theta \varphi_{1}^{\prime}(\mathrm{m}) \cdot \dot{\mathrm{m}}, \\
& \mu_{0}(\dot{\mathrm{h}}+\dot{\mathrm{m}})+\operatorname{curl} \mathrm{e}=0, \\
& \operatorname{curl} \mathrm{h}-\sigma(\theta) \mathrm{e}=0 .
\end{aligned}
$$


For $\Omega$ a domain of $\mathbb{R}^{3}$ and $T>0$ a time, the unknowns are: magnetization $\mathrm{m}:[0, T] \times \Omega \rightarrow \mathbb{R}^{3}$, temperature $\theta:[0, T] \times \Omega \rightarrow \mathbb{R}^{+}$, self-induced magnetic field $\mathrm{h}:[0, T] \times \Omega \rightarrow \mathbb{R}^{3}$, and electric field $\mathrm{e}:[0, T] \times \Omega \rightarrow \mathbb{R}^{3}$. A superposed dot denotes differentiation with respect to time, the symbol " $\times$ " denotes the vector product in $\mathbb{R}^{3}, \lambda>0$ is the exchange constant, $\mu_{0}>0$ is the permeability of vacuum, and $\mathrm{h}_{\mathrm{e}}$ : $[0, T] \times \Omega \rightarrow \mathbb{R}^{3}$ is the applied field. Furthermore, $c(\theta), \kappa(\theta)$ and $\sigma(\theta)$ are, respectively, the heat capacity, the thermal conductivity and the electric conductivity, here allowed to depend on temperature, and required to be strictly positive. The functions $g: \mathbb{R}^{+} \rightarrow \mathbb{R}^{+}, \varphi_{0}: \mathbb{R}^{3} \rightarrow \mathbb{R}, \varphi_{1}: \mathbb{R}^{3} \rightarrow \mathbb{R}$ must comply with certain assumptions that we shall specify later in Sec. 3. Finally, $\varphi_{i}^{\prime}$ stands for the derivative of $\varphi_{i}$.

We complete (2.1) with the boundary conditions:

$$
\begin{gathered}
\partial_{\mathrm{n}} \mathrm{m}=0, \\
\kappa(\theta) \partial_{\mathrm{n}} \theta=\kappa_{\mathrm{b}}\left(\theta_{\mathrm{e}}-\theta\right), \\
\mathrm{h} \times \mathrm{n}=0,
\end{gathered}
$$

and the initial conditions:

$$
\mathrm{m}(0, \cdot)=\mathrm{m}_{0}, \quad \theta(0, \cdot)=\theta_{0}, \quad \mathrm{~h}(0, \cdot)=\mathrm{h}_{0},
$$

where $\partial_{\mathrm{n}}$ denotes the directional derivative along the outward unit normal $\mathrm{n}$ and $\kappa_{\mathrm{b}}$ is a strictly positive constant. Before passing to the weak formulation, several remarks are in order.

Remark 2.1. Equations (2.1a) and (2.1b) are obtained from

$$
\begin{gathered}
\frac{1}{g(|\mathrm{~m}|)} \mathrm{m} \times \dot{\mathrm{m}}+\lambda \Delta \mathrm{m}-\varphi_{0}^{\prime}(\mathrm{m})-\theta \varphi_{1}^{\prime}(\mathrm{m})+\mu_{0}\left(\mathrm{~h}_{\mathrm{e}}+\mathrm{h}\right)-\mathrm{r}=0, \\
c(\theta) \dot{\theta}+\operatorname{div} \mathrm{q}=d+\theta \varphi_{1}^{\prime}(\mathrm{m}) \cdot \dot{\mathrm{m}},
\end{gathered}
$$

a system proposed in Ref. 21 to describe the ferro/paramagnetic transition ${ }^{10}$ that takes place in a homogeneous ferromagnetic body when its temperature approaches the Curie point. A distinguishing feature of this model is the fact that the saturation constraint $|\mathrm{m}|=1$ is dropped, and the underlying free energy

$$
\varphi(\mathrm{m}, \nabla \mathrm{m}, \theta)=\frac{\lambda}{2}|\nabla \mathrm{m}|^{2}+\varphi_{0}(\mathrm{~m})+\theta \varphi_{1}(\mathrm{~m})+\varphi_{\mathrm{t}}(\theta)
$$

contains a Ginzburg-Landau penalization term. The thermal part of the free energy $\varphi_{\mathrm{t}}(\theta)$ determines the heat capacity $c(\theta)=-\theta \varphi_{\mathrm{t}}^{\prime \prime}(\theta)$. In $(2.4 \mathrm{a}), \mathbf{r}$ is a dissipative force associated to the evolution of $m$. Typically, $r$ is taken to be proportional to $\dot{m}$. However, this choice is inappropriate to capture rate-independent dissipation due to pinning effects. Following Ref. 28, we augment the standard viscous-like dissipation with a rate-independent term. Precisely, we require that

$$
r-\alpha \dot{\mathbf{m}} \in \partial \varrho(\dot{\mathrm{m}}),
$$

with $\alpha$ a strictly positive constant and $\varrho$ a convex continuous degree- 1 positively homogeneous function. 
In (2.4b), the scalar field $d:[0, T] \times \Omega \rightarrow \mathbb{R}^{+}$and the vector field $\mathrm{q}:[0, T] \times \Omega \rightarrow$ $\mathbb{R}^{3}$ are, respectively, the dissipation density and the heat flux. We assume that the body carries a conduction current J. Accordingly, the dissipation density is given by the sum of microscopic heating $\mathrm{r} \cdot \dot{\mathrm{m}}$ and Joule heating $\mathrm{j} \cdot \mathrm{e}$ (cf. Ref. 13). We assume that the conduction current satisfies Ohm's law:

$$
\mathrm{J}=\sigma(\theta) \text { e. }
$$

By (2.5) and (2.6), and by the degree-1 homogeneity of $\varrho(\cdot)$, we have:

$$
d=\sigma(\theta)|\mathrm{e}|^{2}+\alpha|\dot{\mathrm{m}}|^{2}+\varrho(\dot{\mathrm{m}}) .
$$

Equation (2.1b) is obtained by combining (2.4b), (2.7), and by assuming that the heat flux depends on the temperature gradient in the manner prescribed by Fourier's law:

$$
\mathbf{q}=-\kappa(\theta) \nabla \theta
$$

Equations (2.1c) and (2.1d) are the eddy-current approximation of Maxwell's equations (cf. Remark 2.4 below), namely:

$$
\begin{gathered}
\mu_{0}(\dot{\mathrm{h}}+\dot{\mathrm{m}})+\operatorname{curl} \mathrm{e}=0, \\
\text { curl } \mathrm{h}-\mathrm{J}=0 .
\end{gathered}
$$

Remark 2.2. According to a well-established phenomenological model first proposed by Landau and Lifshitz, ${ }^{18}$ the evolution of the magnetization in saturated ferromagnets is ruled by the so-called Landau-Lifshitz-Gilbert (LLG) equation ${ }^{5,7,14,18}$ :

$$
\gamma^{-1} \dot{m}+\mathrm{m} \times \mathrm{r}=\mathrm{m} \times\left(\lambda \Delta \mathrm{m}-\varphi^{\prime}(\mathrm{m})+\mathrm{h}\right),
$$

with $\mathbf{r}=\alpha \dot{\mathrm{m}}$. The LLG equation has been the subject of extensive mathematical investigation. ${ }^{2,6,8,9,16,19,27}$ An important property of (2.10) is that it preserves the modulus of the magnetization. In particular, if the initial datum $\mathrm{m}_{0}$ satisfies the saturation constraint $\left|\mathrm{m}_{0}(x)\right|=1$, then so does the solution at all times. The constraint $|\mathrm{m}|=1$ leads to the following (natural) boundary condition for (2.10):

$$
\mathrm{m} \times \partial_{\mathrm{n}} \mathrm{m}=0 .
$$

When the aim of the model is to describe the ferro/paramagnetic transition, the underlying evolution law, that is (2.4a), must allow the modulus of magnetization vector to change both in space and time, and, due to the suppression of the saturation constraint, the boundary condition (2.11) must be replaced by (2.2a). A connection between (2.10) and (2.4a) is established by observing that if $\mathrm{m}$ satisfies (2.4a) with boundary condition (2.2a) and, in addition, it fulfills the saturation condition $|\mathrm{m}|=1$, then it also satisfies (2.10) with boundary condition (2.11). This can be readily seen by noting that $|\mathrm{m}|=1$ implies $\mathrm{m} \cdot \dot{\mathrm{m}}=0$ and by using the identity $\mathrm{m} \times(\mathrm{m} \times \dot{\mathrm{m}})=$ $(\dot{\mathrm{m}} \cdot \mathrm{m}) \mathrm{m}-|\mathrm{m}|^{2} \dot{\mathrm{m}}$.

Remark 2.3. The boundary condition (2.2c) obtains by imposing the standard transmission condition at the boundary: $\left(\mathrm{h}^{+}-\mathrm{h}^{-}\right) \times \mathrm{n}=0$, with superscripts \pm denoting limiting values from the exterior and from the interior of the body, and by 
setting to null the magnetic field outside the body. In Sec. 5, we present a brief explanation of what fails in our proof if one had to consider electric and magnetic fields outside the body. For boundary conditions alternative to $(2.2 \mathrm{c})$, the reader may consult Chap. 7, Sec. 3 of Ref. 11. A consequence of (2.2c) (along with (2.1d)) is that

$$
\sigma(\theta) \mathrm{e} \cdot \mathrm{n}=0
$$

holds on $\partial \Omega$, that is to say, the outward current flux at the boundary vanishes. Indeed, by $(2.1 \mathrm{~d})$ we have $\int_{\Omega}(\operatorname{curl} \mathrm{h}-\sigma(\theta) \mathrm{e}) \cdot \nabla v, \mathrm{~d} x=0$ for all $v \in C^{1}(\bar{\Omega})$. Moreover, from Stokes' formula, and from the boundary condition $(2.2 \mathrm{c})$ it follows that:

$$
\int_{\Omega} \operatorname{curl} \mathrm{h} \cdot \nabla v \mathrm{~d} x=\int_{\Omega} \mathrm{h} \cdot \operatorname{curl} \nabla v \mathrm{~d} x+\int_{\partial \Omega} \mathrm{h} \times \mathrm{n} \cdot \nabla v \mathrm{~d} S=0,
$$

since curl $\nabla v=0$. Thus, $\int_{\Omega} \sigma(\theta) \mathrm{e} \cdot \nabla v \mathrm{~d} x=0$ for all $v \in C^{1}(\bar{\Omega})$, which entails (2.12).

Remark 2.4. System (2.9) is obtained from Maxwell's system

$$
\begin{gathered}
\mu_{0}(\dot{\mathrm{h}}+\dot{\mathrm{m}})+\operatorname{curl} \mathrm{e}=0, \\
\varepsilon \dot{\mathrm{e}}-\operatorname{curl} \mathrm{h}+\mathrm{J}=0,
\end{gathered}
$$

(here $\varepsilon>0$ is the electric permittivity) by neglecting the displacement current $\varepsilon \dot{\mathbf{e}}$. Justifications of the eddy-current approximation when the dependence of all involved fields with respect to time is harmonic may be found in Refs. 1 and 3, where it is shown that the eddy-current approximation can be applied when the characteristic frequencies of the involved fields are small in an appropriate sense. We also remark that from (2.1c) it follows that if the initial conditions are consistent with Gauss' law, that is to say, $\operatorname{div}\left(\mathrm{h}_{0}+\mathrm{m}_{0}\right)=0$ in the sense of distributions, then the solution in $\Omega$ is consistent with Gauss' law at all times.

Remark 2.5. The positivity assumption on $\kappa, \sigma, \alpha$, along with the convexity of $\varrho$, guarantee consistency of (2.5), (2.6), and (2.8) with the Second Law of Thermodynamics, which is locally expressed by the following version of the Reduced Dissipation Inequality ${ }^{13}$ :

$$
0 \leq-\theta^{-1} \mathbf{q} \cdot \nabla \theta+\mathbf{r} \cdot \dot{\mathrm{m}}+\mathrm{J} \cdot \mathrm{e} .
$$

Here we also used positivity of temperature $\theta$, which can indeed be shown under some assumptions, cf. Remark 4.1 below. With only a small loss of generality, we will just consider (cf. also Ref. 22 and references therein):

$$
\varrho(\mathrm{v})=\rho|\mathrm{v}|, \quad \rho>0 .
$$

\section{Weak Formulation, Data Qualification, Existence Result}

We consider the above formulated initial-boundary-value-problem with a finite time horizon $T>0$. We let $I:=(0, T), \Sigma:=I \times \partial \Omega$, and $Q:=I \times \Omega$. We use the standard notation $C^{\infty}(\cdot)$ for the space of smooth (vector- or tensor-valued) functions, $L^{p}(\cdot)$ for $p$-power Lebesgue integrable functions, $W^{k, p}(\cdot)$ for the Sobolev space of functions 
whose $k$ th derivatives are in $L^{p}(\cdot)$, and $\left(W^{k, p}(\cdot)\right)^{*}$ for its dual space. Also, we make use of the Hilbert space $^{15}$ :

$$
L_{\text {curl }, 0}^{2}\left(\Omega ; \mathbb{R}^{3}\right):=\left\{\mathrm{v} \in L^{2}\left(\Omega ; \mathbb{R}^{3}\right) ; \operatorname{curl} \mathbf{v} \in L^{2}\left(\Omega ; \mathbb{R}^{3}\right), \mathrm{v} \times\left.\mathrm{n}\right|_{\partial \Omega}=0\right\} .
$$

Moreover, for $X$ a Banach space, we denote by $L^{p}(I ; X)$ the $L^{p}$-Bochner space of $X$-valued functions, by $W^{k, p}(I ; X)$ the corresponding Sobolev-Bochner space, and by $\mathcal{M}(\bar{I} ; X)$ the space of $X$-valued measures on $\bar{I}=[0, T]$. The scalar product of vectors from $\mathbb{R}^{3}$ and the matrices from $\mathbb{R}^{3 \times 3}$ will be denoted by "." and ":", respectively. As usual, $p^{\prime}=p /(p-1)$.

We make the following assumptions:

$g, \kappa: \mathbb{R}^{+} \rightarrow \mathbb{R}^{+}$continuous,

$c, \sigma: \mathbb{R}^{+} \rightarrow \mathbb{R}^{+}, \quad \varphi_{0}, \varphi_{1}: \mathbb{R}^{3} \rightarrow \mathbb{R}$ continuously differentiable,

$\exists g_{0}, g_{1}>0: g(m) \geq g_{0}+g_{1} m$,

$\exists c_{\max } \geq c_{\min }>0, \omega>3 / 2: c_{\min }\left(1+\theta^{\omega-1}\right) \leq c(\theta) \leq c_{\max }\left(1+\theta^{\omega-1}\right)$,

$\exists \kappa_{\max } \geq \kappa_{\min }>0: \kappa_{\min } \leq \kappa(\theta) \leq \kappa_{\max }$

$\exists \sigma_{\max } \geq \sigma_{\min }>0: \sigma_{\min } \leq \sigma(\theta) \leq \sigma_{\max }, \quad\left|\sigma^{\prime}(\theta)\right| \leq \sigma_{\max }$,

$\exists \zeta>0, \quad C_{\max } \in \mathbb{R}: c^{\prime}(\theta) \leq C_{\max } \frac{c(\theta)^{2}}{(1+\theta)^{1+\zeta}}$,

$\exists 1 \leq q \leq 4, \quad C_{\min }>0: C_{\min }|\mathrm{m}|^{q} \leq \varphi_{0}(\mathrm{~m})$,

$\exists q_{0}<3, \quad C_{\max } \in \mathbb{R}:\left|\varphi_{0}^{\prime}(\mathrm{m})\right| \leq C_{\max }\left(1+|\mathrm{m}|^{q_{0}}\right)$,

$\exists q_{1}>\min \left(\frac{2 \omega}{\omega-2}, \frac{6 \omega}{2 \omega-3}\right), \quad C_{\max } \in \mathbb{R}:\left|\varphi_{1}^{\prime}(\mathrm{m})\right|^{q_{1}}<C_{\max }\left(1+\varphi_{0}(\mathrm{~m})\right)$,

$\mathrm{h}_{\mathrm{e}} \in W^{1,1}\left(I ; L^{2}\left(\Omega ; \mathbb{R}^{3}\right)\right)$,

$\theta_{\mathrm{e}} \in L^{1}(\Sigma), \quad \theta_{\mathrm{e}} \geq 0$,

$\mathrm{m}_{0} \in W^{1,2}\left(\Omega ; \mathbb{R}^{3}\right), \quad \theta_{0} \in L^{\omega}(\Omega), \quad \mathrm{e}_{0}, \mathrm{~h}_{0} \in L^{2}\left(\Omega ; \mathbb{R}^{3}\right), \quad \theta_{0} \geq 0$.

Definition 3.1. (Weak solutions) Let

$$
\begin{aligned}
& \mathrm{m} \in W^{1,2}\left(I ; L^{2}\left(\Omega ; \mathbb{R}^{3}\right)\right) \cap L^{\infty}\left(I ; W^{1,2}\left(\Omega ; \mathbb{R}^{3}\right)\right), \\
& \theta \in L^{r}\left(I ; W^{1, r}(\Omega)\right) \cap L^{\infty}\left(I ; L^{\omega}(\Omega)\right) \quad \text { with } r \in\left[1, \frac{2 \omega+3}{\omega+3}\right), \\
& \mathrm{h} \in L^{\infty}\left(I ; L^{2}\left(\Omega ; \mathbb{R}^{3}\right)\right), \\
& \mathrm{e} \in L^{2}\left(Q ; \mathbb{R}^{3}\right),
\end{aligned}
$$

with $\theta \geq 0$ a.e. in $Q$. We say that the quadruple $(\mathrm{m}, \theta, \mathrm{h}, \mathrm{e})$ is a weak solution to system (2.1) with boundary conditions (2.2) and initial conditions (2.3) if:

(i) $\mathrm{m}(0, \cdot)=\mathrm{m}_{0}$ and there exists $\mathrm{r} \in L^{2}\left(Q ; \mathbb{R}^{3}\right)$ such that for a.a. $t \in I$ and for all $\mathbf{z} \in W^{1,2}\left(\Omega ; \mathbb{R}^{3}\right)$ and $\mathbf{w} \in L^{2}\left(\Omega ; \mathbb{R}^{3}\right)$,

$$
\begin{aligned}
\int_{\Omega} \mathrm{r} \cdot \mathbf{z} & +\lambda \nabla \mathrm{m}: \nabla \mathbf{z}+\varphi_{0}^{\prime}(\mathrm{m}) \cdot \mathbf{z d} x \\
= & \int_{\Omega} \frac{\mathrm{m} \times \dot{\mathrm{m}}}{g(|\mathrm{~m}|)} \cdot \mathbf{z}-\theta \varphi_{1}^{\prime}(\mathrm{m}) \cdot \mathbf{z}+\mu_{0}\left(\mathrm{~h}+\mathrm{h}_{\mathrm{e}}\right) \cdot \mathbf{z d} x,
\end{aligned}
$$




$$
\int_{\Omega} \varrho(\mathbf{w})-\varrho(\dot{\mathbf{m}})-(\mathbf{r}-\alpha \dot{\mathbf{m}}) \cdot(\mathbf{w}-\dot{\mathrm{m}}) \mathrm{d} x \geq 0 ;
$$

(ii) for all $z \in C^{1}(\bar{Q})$ such that $z(T, \cdot)=0$,

$$
\begin{aligned}
\int_{Q}-\hat{c}(\theta) \cdot \dot{\mathrm{z}}+\kappa(\theta) \nabla \theta \cdot \nabla z \mathrm{~d} x \mathrm{~d} t \\
=\int_{Q}\left(\sigma(\theta)|\mathrm{e}|^{2}+\mathrm{r} \cdot \dot{\mathrm{m}}+\theta \varphi_{1}^{\prime}(\mathrm{m}) \cdot \dot{\mathrm{m}}\right) z \mathrm{~d} x \mathrm{~d} t \\
\quad+\int_{\Sigma} \kappa_{\mathrm{b}}\left(\theta_{\mathrm{e}}-\theta\right) z \mathrm{~d} S \mathrm{~d} t+\int_{\Omega} \hat{c}\left(\theta_{0}\right) z(0, \cdot) \mathrm{d} x
\end{aligned}
$$

where $\hat{c}(\cdot)$ is a primitive of $c(\cdot)$;

(iii) for all $\mathrm{u} \in C^{1}\left(\bar{I} ; L^{2}\left(\Omega ; \mathbb{R}^{3}\right)\right) \cap C\left(\bar{I} ; L_{\text {curl, } 0}^{2}\left(\Omega ; \mathbb{R}^{3}\right)\right)$ such that $\mathrm{u}(T, \cdot)=0$

$$
\int_{Q} \mathrm{e} \cdot \operatorname{curl} \mathrm{u}-\mu_{0}(\mathrm{~h}+\mathrm{m}) \cdot \dot{\mathrm{u}} \mathrm{d} x \mathrm{~d} t=\int_{\Omega} \mu_{0}\left(\mathrm{~h}_{0}+\mathrm{m}_{0}\right) \cdot \mathrm{u}(0, \cdot) \mathrm{d} x ;
$$

(iv) for all $\mathbf{v} \in C\left(\bar{I} ; W^{1,2}\left(\Omega ; \mathbb{R}^{3}\right)\right)$

$$
\int_{Q} \mathrm{~h} \cdot \operatorname{curl} \mathrm{v}-\sigma(\theta) \mathrm{e} \cdot \mathrm{vd} x \mathrm{~d} t=0 .
$$

Note that the above definition reflects that, in contrast to $\mathrm{m}$, the time derivative of $\theta$ and $\mathrm{h}$ is estimated only poorly, cf. (3.7), and thus, in contrast to (3.3), the by-part integration has been employed for (3.4) and (3.5), while e has no time derivative considered at all; in fact, e.g. if $\sigma$ is constant, then we would have $\dot{\mathrm{e}}=\frac{1}{\sigma}$ curl $\dot{\mathrm{h}} \in$ $L^{2}\left(I ; W^{2,2}\left(\Omega ; \mathbb{R}^{3}\right)^{*}\right)$. We also note that from the boundedness of $\sigma(\cdot)$ imposed by (3.2f) and from (3.6) it follows that $\mathrm{h} \in L^{2}\left(I ; L_{\text {curl }, 0}^{2}\left(\Omega ; \mathbb{R}^{3}\right)\right)$.

Now we state the analytical result whose proof will be given in Sec. 4:

Theorem 3.1. (Existence of weak solutions) Under the assumptions (3.2), the initial-boundary-value problem (2.1)-(2.3) admits at least one weak solution $(\mathrm{m}, \theta$, $\mathrm{h}, \mathrm{e})$ in accord to Definition 3.1. Moreover, $\theta \geq 0$ and

$$
\begin{gathered}
\dot{\theta} \in \mathcal{M}\left(I ; W^{3,2}(\Omega)^{*}\right), \\
(\hat{c}(\theta))^{\cdot} \in L^{1}\left(I ; W^{3,2}(\Omega)^{*}\right), \\
\dot{\mathrm{h}} \in L^{2}\left(I ; L_{\text {curl }, 0}^{2}\left(\Omega ; \mathbb{R}^{3}\right)^{*}\right) .
\end{gathered}
$$

The proof of this theorem is performed by a quite constructive way in the following Sec. 4, in particular it follows from Proposition 4.2, and the estimates (3.7a) and (3.7b) follows as in Proposition 3.10 of Ref. 21 while the estimate (3.7c) is due to the Eq. (2.1c) and the a priori estimates (4.21a) and (4.21c).

\section{Proof of Existence of Weak Solutions}

In our proofs, positive constants dependent only on the data will be denoted by $C$. When two or more constants appear in the same formula, they will be denoted by a 
progressive integer subscript $\left(C_{1}, C_{2}\right.$, etc.). We will prove Theorem 3.1 by several carefully assembled steps:

(A) we modify the original problem by a suitable extension for $\theta<0$ and then we build a discrete Galerkin approximation of the modified problem. We use the index $k \in \mathbb{N}$ to enumerate the Galerkin approximation steps for the unknowns $\mathrm{m}$, $\mathrm{h}$ and $\mathrm{e}$, and the index $l \in \mathbb{N}$ to enumerate the Galerkin approximation steps for $\theta$. Moreover, we replace dry friction with a smooth viscosity by approximating $\varrho$ with smooth convex functions $\varrho_{n}$. We also use the index $n$ to enumerate a sequence of monotone terms having a $p$-growth to compensate the growth of the non-monotone terms. By doing so, we can prove existence of an approximate solution in the time interval $[0, T]$ by using standard existence theory for ordinary differential equations (or rather differential-algebraic equations), a priori estimates, and prolongation arguments (cf. the proof of Lemma 4.1);

(B) we let $l \rightarrow+\infty$, thus passing to the limit with respect to the Galerkin discretization for $\theta$ in the heat equation, which allows for proving non-negativity of the approximated temperature, cf. the proofs of Lemmas 4.1 and 4.2;

(C) we perform the physically relevant a priori estimates (i.e. "real" energy bounds), cf. (4.19a)-(4.19f);

(D) using the $L^{1}$-theory for heat equation together with Gagliardo-Nirenberg interpolation made simultaneously for the thermal and magnetic parts, we derive estimate on the temperature gradient, cf. (4.21a), and estimates on dissipation, cf. (4.21b) and (4.21c);

(E) we let $n \rightarrow+\infty$ and we show that $\varrho_{n}^{\prime}\left(\dot{\mathrm{m}}_{k}\right)+\alpha \dot{\mathrm{m}}_{k} \rightarrow \mathrm{r}_{k}$ weakly in $L^{2}\left(Q ; \mathbb{R}^{3}\right)$, where $\mathbf{r}_{k}$ satisfies the differential inequality (3.3b) with $\mathrm{m}=\mathrm{m}_{k}$, cf. Lemma 4.4 and in particular $(4.47 \mathrm{~b})$;

(F) we perform the final limit passage by letting $k \rightarrow+\infty$, cf. Proposition 4.2.

To perform the Galerkin procedure, let us take an increasing sequence $\left\{V_{k}\right\}_{k \in \mathbb{N}}$ of finite-dimensional subspaces of $W^{1, \infty}(\Omega)$ such that $\cup_{k \in \mathbb{N}} V_{k}$ is dense in $W^{1,2}(\Omega)$. We approximate $\dot{\mathrm{m}}$ with elements from $\left\{V_{k}^{3}\right\}_{k \in \mathbb{N}}$, and $\theta$ with elements from $\left\{V_{k}\right\}_{k \in \mathbb{N}}$. Note that $\cup_{k \in \mathbb{N}} V_{k}$ is therefore also dense in $L^{\omega}(\Omega)$. Moreover, we take an increasing sequence $\left\{U_{k}\right\}_{k \in \mathbb{N}}$ of finite-dimensional subspaces of $W^{1, \infty}\left(\Omega ; \mathbb{R}^{3}\right)$ such that $\mathrm{h}_{k} \times \mathbf{n}=0$ on $\partial \Omega$ for all $\mathrm{h}_{k} \in U_{k}$ and $\left\{U_{k}\right\}_{k \in \mathbb{N}}$ is dense in $L_{\text {curl, } 0}^{2}\left(\Omega ; \mathbb{R}^{3}\right)$. This choice of the basis guarantees that the following "curl-cancellation" property holds:

$$
\int_{\Omega} \operatorname{curl} \mathrm{h} \cdot \mathrm{e}-\mathrm{h} \cdot \operatorname{curl} \mathrm{ed} x=0 \quad \forall(\mathrm{h}, \mathrm{e}) \in U_{k} \times V_{k}^{3} .
$$

Furthermore, we consider an approximation $\varrho_{n}$ of $\varrho$ satisfying, for $n \rightarrow \infty$,

$$
\begin{aligned}
& \varrho_{n}: \mathbb{R}^{3} \rightarrow \mathbb{R}^{+} \quad \text { is a convex } C_{1} \text {-function with } \varrho_{n}^{\prime}: \mathbb{R}^{3} \rightarrow \mathbb{R}^{3} \text { bounded, } \\
& \varrho_{n}^{\prime}(\mathrm{m}) \cdot \mathrm{m} \geq 0, \quad \varrho_{n}^{\prime}(0)=0 \\
& \varrho_{n} \rightarrow \varrho \quad \text { uniformly on } \mathbb{R}^{3},
\end{aligned}
$$


$\varrho_{n}^{\prime} \rightarrow \varrho^{\prime} \quad$ pointwise on $\mathbb{R}^{3} \backslash\{0\}, \quad$ and

$\tilde{\varrho}_{n} \rightarrow \varrho$ uniformly on $\mathbb{R}^{3}$ with $\varrho_{n}(\mathrm{~m}):=\mathrm{m} \varrho_{n}^{\prime}(\mathrm{m})$.

This is satisfied e.g. by the Yosida approximation of $\varrho$ from (2.15) or just by taking $\varrho_{n}(\mathrm{~m}):=\rho n|\mathrm{~m}|^{3} /\left(n|\mathrm{~m}|^{2}+1\right)$.

The following lemma states the existence of a solution to a semi-discrete regularized system, namely Galerkin approximation for Gilbert's and Maxwell's equations, and continuous version of the heat equation, while the full Galerkin approximation advertised in (A) is "hidden" in its proof.

We also approximate the initial data $\mathrm{m}_{0}, \theta_{0}$, and $\mathrm{h}_{0}$ and the boundary datum $\theta_{\mathrm{e}}$ by choosing appropriate sequences $\mathrm{m}_{0, k}, \theta_{0, k}, \mathrm{~h}_{0, k}$, and $\theta_{\mathrm{e}, k}$ such that

$$
\begin{aligned}
& \mathrm{m}_{0, k} \in V_{k}^{3} \quad \text { and } \quad \lim _{k \rightarrow \infty} \mathrm{m}_{0, k}=\mathrm{m}_{0} \quad \text { in } W^{1,2}\left(\Omega ; \mathbb{R}^{3}\right) \text {, } \\
& \theta_{0, k} \in V_{k} \quad \text { and } \quad \lim _{k \rightarrow \infty} \theta_{0, k}=\theta_{0} \quad \text { in } L^{\omega}(\Omega) \text {, } \\
& \mathrm{h}_{0, k} \in U_{k} \quad \text { and } \quad \lim _{k \rightarrow \infty} \mathrm{h}_{0, k}=\mathrm{h}_{0} \quad \text { in } L_{\text {curl, } 0}^{2}\left(\Omega ; \mathbb{R}^{3}\right) \text {, } \\
& \theta_{\mathrm{e}, k} \in W^{1,1}\left(I ; L^{\infty}(\Gamma)\right) \text { and } \quad \lim _{k \rightarrow \infty} \theta_{\mathrm{e}, k}=\theta_{\mathrm{e}} \quad \text { in } L^{1}(\Sigma) .
\end{aligned}
$$

The approximation of $\theta_{\mathrm{e}}$ and $\theta_{0}$ will allow, in particular, for usage of the conventional $L^{2}$-theory for the approximated heat equation.

Lemma 4.1. (Regularization and semi-Galerkin approximation) Let the exponent $p$ be chosen large enough to satisfy

$$
p>\frac{2(1+\omega)}{\omega}, \quad \frac{1}{1+\omega}+\frac{1}{q_{1}}+\frac{1}{p}<1 .
$$

Let also the function $c(\cdot)$ be extended to negative arguments by defining $c(\theta)=c(-\theta)$ for all $\theta<0$. Then, there exist $\mathrm{m}_{k n} \in W^{1,2}\left(I ; V_{k}^{3}\right), \quad \theta_{k n} \in L^{2}\left(I ; W^{1,2}(\Omega)\right) \cap$ $W^{1,1}\left(I ; L^{2}(\Omega)\right), \mathrm{e}_{k n} \in L^{p}\left(I ; V_{k}^{3}\right)$, and $\mathrm{h}_{k n} \in W^{1, p}\left(I ; U_{k}\right)$ such that

$$
\begin{gathered}
\int_{\Omega}\left(\alpha \dot{\mathrm{m}}_{k n}+\varrho_{n}^{\prime}\left(\dot{\mathrm{m}}_{k n}\right)+\varphi_{0}^{\prime}\left(\mathrm{m}_{k n}\right)+\frac{1}{n}\left|\dot{\mathrm{m}}_{k n}\right|^{p-2} \dot{\mathrm{m}}_{k n}\right) \cdot \mathbf{z}+\lambda \nabla \mathbf{m}_{k n}: \nabla \mathbf{z d} x \\
=\int_{\Omega}\left(\frac{\mathrm{m}_{k n} \times \dot{\mathrm{m}}_{k n}}{g\left(\left|\mathrm{~m}_{k n}\right|\right)}-\theta_{k n}^{+} \varphi_{1}^{\prime}\left(\mathrm{m}_{k n}\right)+\mu_{0} \mathrm{~h}_{k n}+\mu_{0} \mathrm{~h}_{\mathrm{e}}\right) \cdot \mathbf{z d} x \\
\int_{\Omega} c\left(\theta_{k n}\right) \dot{\theta}_{k n} z+\kappa\left(\theta_{k n}\right) \nabla \theta_{k n} \cdot \nabla z \mathrm{~d} x \\
=\int_{\Omega}\left(\sigma\left(\theta_{k n}\right)\left|\mathbf{e}_{k n}\right|^{2}+\alpha\left|\dot{\mathrm{m}}_{k n}\right|^{2}+\varrho_{n}^{\prime}\left(\dot{\mathrm{m}}_{k n}\right) \cdot \dot{\mathrm{m}}_{k n}+\theta_{k n}^{+} \varphi_{1}^{\prime}\left(\mathrm{m}_{k n}\right) \cdot \dot{\mathrm{m}}_{k n}\right) z \\
+\int_{\partial \Omega} \kappa_{\mathrm{b}}\left(\theta_{\mathrm{e}, k}-\theta_{k n}\right) z \mathrm{~d} S, \\
\int_{\Omega} \mu_{0} \dot{\mathrm{h}}_{k n} \cdot \mathrm{u}+\mathrm{e}_{k n} \cdot \operatorname{curl} \mathbf{u}+\mu_{0} \dot{\mathrm{m}}_{k n} \cdot \mathrm{ud} x=0, \\
\int_{\Omega}\left(\frac{1}{n}\left|\mathbf{e}_{k n}\right|^{p-2} \mathbf{e}_{k n}+\sigma\left(\theta_{k n}\right) \mathbf{e}_{k n}\right) \cdot \mathrm{v}-\mathrm{h}_{k n} \cdot \operatorname{curl} \mathrm{vd} x=0
\end{gathered}
$$


for all $\mathrm{z}, \mathrm{v} \in V_{k}^{3}, \mathrm{u} \in U_{k}$, and $z \in W^{1,2}(\Omega)$ and for a.a. $t \in I$, and satisfying the initial conditions

$$
\mathrm{m}_{k n}(0, \cdot)=\mathrm{m}_{0, k}, \quad \theta_{k n}(0, \cdot)=\theta_{0, k}, \quad \mathrm{~h}_{k n}(0, \cdot)=\mathrm{h}_{0, k} .
$$

Sketch of the Proof. We use essentially the technique from Ref. 21 and thus we present only main steps and modifications.

We still perform the Galerkin approximation of (4.5b) by using the finitedimensional space $V_{l}, l \in \mathbb{N}, l \geq k$. Thus we obtain the initial-value problem for a system of ordinary differential-algebraic equations, let us denote its solution by $\left(\mathrm{m}_{k n l}, \theta_{k n l}, \mathrm{~h}_{k n l}, \mathrm{e}_{k n l}\right)$. More precisely, we can see the so-called underlying ODE-system by differentiating $(4.5 \mathrm{c})$ once in time and combining it with (2.1c), which leads formally to

$$
\left(\sigma\left(\theta_{k n l}\right)+(p-2)\left|\mathbf{e}_{k n l}\right|^{p-2}\right) \dot{\mathbf{e}}_{k n l}+\sigma^{\prime}\left(\theta_{k n l}\right) \dot{\theta}_{k n l}+\dot{\mathrm{m}}_{k n l}+\frac{1}{\mu_{0}} \operatorname{curl}^{2} \mathbf{e}_{k n l} \approx 0
$$

in the Galerkin scheme, the right-hand side of (4.7) is not zero but just the residuum orthogonal to $V_{k}$. Existence of such a solution then follows by standard arguments: first one can show this locally, and then successively prolong when using the $L^{\infty}(I)$-estimates. These estimates can be obtained by testing the equations respectively by $\dot{\mathrm{m}}_{k n l}, \theta_{k n l}, \mathrm{~h}_{k n l}$ and $\mathrm{e}_{k n l}$. Let us introduce the auxiliary potential $\mathfrak{C}: \mathbb{R} \rightarrow \mathbb{R}$ defined by $\mathfrak{C}(\theta):=\int_{0}^{\theta} \vartheta c(\vartheta) \mathrm{d} \vartheta$. Note that $\theta c(\theta) \dot{\theta}=(\mathfrak{C}(\theta))^{\bullet}$ and that $(3.2 \mathrm{~d})$ ensures

$$
c_{\min }\left(\frac{1}{2} \theta^{2}+\frac{1}{1+\omega}|\theta|^{1+\omega}\right) \leq \mathfrak{C}(\theta) \leq c_{\max }\left(\frac{1}{2} \theta^{2}+\frac{1}{1+\omega}|\theta|^{1+\omega}\right) .
$$

Note also that $p$ satisfying (4.4) can be chosen by virtue of (3.2j). The above-mentioned test gives, after summation and by using the curl-cancellation $\int_{\Omega} \operatorname{curl} \mathrm{e}_{k n l} \cdot \mathrm{h}_{k n l}-$ $\operatorname{curl}_{k n l} \cdot \mathrm{e}_{k n l} \mathrm{~d} x=0$, the following identity:

$$
\begin{aligned}
\frac{\mathrm{d}}{\mathrm{d} t} \int_{\Omega} & \frac{\lambda}{2}\left|\nabla \mathrm{m}_{k n l}\right|^{2}+\varphi_{0}\left(\mathrm{~m}_{k n l}\right)+\mathfrak{C}\left(\theta_{k n l}\right)+\frac{\mu_{0}}{2}\left|\mathrm{~h}_{k n l}\right|^{2} \mathrm{~d} x \\
& +\int_{\Omega} \alpha\left|\dot{\mathrm{m}}_{k n l}\right|^{2}+\varrho_{n}^{\prime}\left(\dot{\mathrm{m}}_{k n l}\right) \cdot \dot{\mathrm{m}}_{k n l}+\frac{1}{n}\left|\dot{\mathrm{m}}_{k n l}\right|^{p}+\frac{1}{n}\left|\mathrm{e}_{k n}\right|^{p} \\
& +\kappa\left(\theta_{k n l}\right)\left|\nabla \theta_{k n l}\right|^{2}+\sigma\left(\theta_{k n l}\right)\left|\mathrm{e}_{k n l}\right|^{2} \mathrm{~d} x+\int_{\partial \Omega} \kappa_{\mathrm{b}} \theta_{k n l}^{2} \mathrm{~d} S \\
= & \int_{\Omega} \alpha\left|\dot{\mathrm{m}}_{k n l}\right|^{2} \theta_{k n l}+\varrho_{n}\left(\dot{\mathrm{m}}_{k n l}\right) \theta_{k n l}+\left(\theta_{k n l}^{+}\right)^{2} \varphi_{1}^{\prime}\left(\mathrm{m}_{k n l}\right) \cdot \dot{\mathrm{m}}_{k n l} \\
& -\theta_{k n l}^{+} \varphi_{1}^{\prime}\left(\mathrm{m}_{k n l}\right) \cdot \dot{\mathrm{m}}_{k n l}+\mu_{0} \dot{\mathrm{m}}_{k n l} \cdot \mathrm{h}_{k n l}+\sigma\left(\theta_{k n l}\right)\left|\mathrm{e}_{k n l}\right|^{2} \theta_{k n l} \\
& +\mu_{0} \dot{\mathrm{m}}_{k n l} \cdot \mathrm{h}_{\mathrm{e}} \mathrm{d} x+\int_{\partial \Omega} \kappa_{\mathrm{b}} \theta_{\mathrm{e}, k} \theta_{k n l} \mathrm{~d} S .
\end{aligned}
$$

The terms on the right-hand side of (4.9) are to be treated by the Hölder, Young and Gronwall inequalities. The difficult terms are $\alpha\left|\dot{\mathrm{m}}_{k n l}\right|^{2} \theta_{k n l}$ and $\theta_{k n l}^{2} \varphi_{1}^{\prime}\left(\mathrm{m}_{k n l}\right) \cdot \dot{\mathrm{m}}_{k n l}$ and 
they require the regularizing term in the Gilbert equation with $p$ considered sufficiently large as in (4.4), cf. Ref. 21 for details. Another difficult term is $\sigma\left(\theta_{k n l}\right)\left|\mathrm{e}_{k n l}\right|^{2} \theta_{k n l}$ which can be estimated and then handled by the Gronwall inequality due to the regularizing term in the Maxwell equation as

$$
\begin{aligned}
\int_{\Omega} \sigma\left(\theta_{k n l}\right)\left|\mathbf{e}_{k n l}\right|^{2} \theta_{k n l} \mathrm{~d} x & \leq \sigma_{\max }\left\|\left|\mathbf{e}_{k n l}\right|^{2}\right\|_{L^{1+1 / \omega}(\Omega)}\left\|\theta_{k n l}\right\|_{L^{1+\omega}(\Omega)} \\
& =\sigma_{\max }\left\|\mathbf{e}_{k n l}\right\|_{L^{2+2 / \omega}\left(\Omega ; \mathbb{R}^{3}\right)}^{2}\left\|\theta_{k n l}\right\|_{L^{1+\omega}(\Omega)} \\
& \leq \frac{1}{2 n}\left\|\mathbf{e}_{k n l}\right\|_{L^{p}\left(\Omega ; \mathbb{R}^{3}\right)}^{p}+C_{\omega, n, \sigma_{\max }}\left\|\theta_{k n l}\right\|_{L^{1+\omega}(\Omega)}^{1+\omega}
\end{aligned}
$$

provided $p \geq 2+2 / \omega$, as we indeed assumed in (4.4). All these terms make the obtained estimates $n$-dependent. The other terms have lesser growth and can thus be treated more easily and we thus skip the details. Altogether, we thus obtain the estimates

$$
\begin{gathered}
\left\|\mathrm{m}_{k n l}\right\|_{W^{1,2}\left(I ; W^{1,2}\left(\Omega ; \mathbb{R}^{3}\right)\right) \cap L^{\infty}\left(I ; L^{q}\left(\Omega ; \mathbb{R}^{3}\right)\right)} \leq C_{1, k n}, \\
\left\|\dot{\mathrm{m}}_{k n l}\right\|_{L^{p}\left(Q ; \mathbb{R}^{3}\right)} \leq C_{2, k n}, \\
\left\|\theta_{k n l}\right\|_{L^{\infty}\left(I ; L^{1+\omega}(\Omega)\right) \cap L^{2}\left(I ; W^{1,2}(\Omega)\right)} \leq C_{3, k n}, \\
\left\|\mathrm{e}_{k n l}\right\|_{L^{p}\left(Q ; \mathbb{R}^{3}\right)} \leq C_{4, k n}, \\
\left\|\mathrm{~h}_{k n l}\right\|_{L^{\infty}\left(I ; L^{2}\left(\Omega ; \mathbb{R}^{3}\right)\right)} \leq C_{5, k n} .
\end{gathered}
$$

We still need some estimate on $\dot{\theta}_{k n l}$, which can be obtained by testing the Gilbert equation by $\dot{\mathrm{m}}_{k n l}$ and the heat equation by $\dot{\theta}_{k n l}$; for details see Lemma 3.6 of Ref. 21 and realize the estimate for the additional term $\int_{\Omega} \sigma\left(\theta_{k n l}\right)\left|\mathrm{e}_{k n l}\right|^{2} \dot{\theta}_{k n l} \mathrm{~d} t \leq C_{\omega, n, \sigma_{\max }}+$ $\frac{1}{2 n}\left\|\mathrm{e}_{k n l}\right\|_{L^{p}\left(\Omega ; \mathbb{R}^{3}\right)}^{p}+\frac{c_{\min }}{2}\left\|\dot{\theta}_{k n l}\right\|_{L^{2}(\Omega)}^{2}$ provided $p>2+2 / \omega$. Thus we obtain

$$
\left\|\dot{\theta}_{k n l}\right\|_{L^{1}\left(I ; V_{k}^{*}\right)} \leq C_{6, k n}
$$

These a priori estimates then allow for the limit passage for $l \rightarrow \infty$ when a converging subsequence of $\left\{\left(\mathrm{m}_{k n l}, \theta_{k n l}, \mathrm{~h}_{k n l}, \mathrm{e}_{k n l}\right)\right\}_{l \in \mathbb{N}}$ is selected, benefiting from the fact that $V_{k}$ is kept finite-dimensional, so we have strong convergence in $\mathrm{m}_{k n l}$. In particular, $\mathrm{m}_{k n l} \rightarrow$ $\mathrm{m}_{k n}$ strongly in $L^{\infty}\left(Q ; \mathbb{R}^{3}\right)$ and then also

$$
\varphi_{i}^{\prime}\left(\mathrm{m}_{k n l}\right) \rightarrow \varphi_{i}^{\prime}\left(\mathrm{m}_{k n}\right) \quad \text { strongly in } L^{\infty}\left(Q ; \mathbb{R}^{3}\right) \quad \text { for } i=1,2 .
$$

This result allows us to cope with the nonlinearity on the right-hand side of the discretized version of (3.3a).

Further, we have $-\left(\operatorname{curl} \mathrm{e}_{k n l}+\dot{\mathrm{m}}_{k n l}\right) / \mu_{0} \in L^{p}\left(I ; V_{k}^{3}\right)$, hence $\dot{\mathrm{h}}_{k n l} \in L^{p}\left(I ; U_{k}^{*}\right)$. The passage to the limit in $\mathrm{h}_{k n l}$ is easy because it appears linearly. In order to pass to the limit in the discretized versions of (3.4) and (3.6), we need to establish strong convergence of $\theta_{k n l}$. The $L^{2}\left(I ; W^{1,2}(\Omega)\right)$ bound for $\theta_{k n l}$ given by $(4.11 \mathrm{c})$, the $L^{1}\left(I ; V_{K}^{*}\right)$ bound for $\dot{\theta}_{k n l}$ given by (4.12), and a generalization of the Aubin-Lions lemma to locally-convex-space valued time derivatives (cf. Ref. 24, Lemma 7.7) imply that

$$
\theta_{k n l} \rightarrow \theta_{k n} \quad \text { strongly in } L^{2}\left(I ; L^{6-\epsilon}(\Omega)\right)
$$


for every sufficiently small $\epsilon>0$. By standard interpolation results (cf. Lemma 7.8 of Ref. 24), the strong convergence (4.14) combined with the $L^{\infty}\left(I ; L^{1+\omega}(\Omega)\right)$ bound provided by (4.11c) yields the "isotropic" estimate (cf. Ref. 21, Eq. (3.32)):

$$
\theta_{k n l} \rightarrow \theta_{k n} \quad \text { strongly in } L^{(8+2 \omega) / 3-\epsilon}(Q)
$$

for every sufficiently small $\epsilon>0$. For limit passage in the heat equation, it is also important to have strong convergence of $\dot{\mathrm{m}}_{k n l} \rightarrow \dot{\mathrm{m}}_{k n}$ and of $\mathrm{e}_{k n l} \rightarrow \mathrm{e}_{k n}$ in $L^{2}\left(Q ; \mathbb{R}^{3}\right)$.

The former convergence can be obtained by testing the discretized Gilbert equation by $\dot{\mathrm{m}}_{k n l}-\dot{\mathrm{m}}_{k n}$ which is a legal test since the space $V_{k}^{3}$ is the same for all $l$. This gives

$$
\begin{aligned}
\alpha \| \dot{\mathrm{m}}_{k n l} & -\dot{\mathrm{m}}_{k n}\left\|_{L^{2}\left(Q ; \mathbb{R}^{3}\right)}^{2}+\frac{c_{p}}{n}\right\| \dot{\mathrm{m}}_{k n l}-\dot{\mathrm{m}}_{k n} \|_{L^{p}\left(Q ; \mathbb{R}^{3}\right)}^{p} \\
\leq & \alpha\left\|\dot{\mathrm{m}}_{k n l}-\dot{\mathrm{m}}_{k n}\right\|_{L^{2}\left(Q ; \mathbb{R}^{3}\right)}^{2}+\int_{Q}\left(\varrho_{n}^{\prime}\left(\dot{\mathrm{m}}_{k n l}\right)+\frac{1}{n}\left|\dot{\mathrm{m}}_{k n l}\right|^{p-2} \dot{\mathrm{m}}_{k n l}\right. \\
& \left.-\varrho_{n}^{\prime}\left(\dot{\mathrm{m}}_{k n}\right)-\frac{1}{n}\left|\dot{\mathrm{m}}_{k n}\right|^{p-2} \dot{\mathrm{m}}_{k n}\right) \cdot\left(\dot{\mathrm{m}}_{k n l}-\dot{\mathrm{m}}_{k n}\right) \mathrm{d} x \mathrm{~d} t \\
\leq & \int_{Q}\left|\varphi_{0}^{\prime}\left(\mathrm{m}_{k n l}\right) \cdot\left(\dot{\mathrm{m}}_{k n l}-\dot{\mathrm{m}}_{k n}\right)\right| \mathrm{d} x \mathrm{~d} t \\
& +\int_{Q}\left|\left(\alpha \dot{\mathrm{m}}_{k n}+\frac{1}{n}\left|\dot{\mathrm{m}}_{k n}\right|^{p-2} \dot{\mathrm{m}}_{k n}+\varrho_{n}^{\prime}\left(\dot{\mathrm{m}}_{k n}\right)\right) \cdot\left(\dot{\mathrm{m}}_{k n l}-\dot{\mathrm{m}}_{k n}\right)\right| \mathrm{d} x \mathrm{~d} t \\
& +\int_{Q}\left|\theta_{k n l} \varphi_{1}^{\prime}\left(\mathrm{m}_{k n l}\right) \cdot\left(\dot{\mathrm{m}}_{k n l}-\dot{\mathrm{m}}_{k n}\right)\right| \mathrm{d} x \mathrm{~d} t \\
& +\int_{Q}\left|\frac{\mathrm{m}_{k n l}}{g\left(\left|\mathrm{~m}_{k n l}\right|\right)} \times \dot{\mathrm{m}}_{k n l} \cdot\left(\dot{\mathrm{m}}_{k n l}-\dot{\mathrm{m}}_{k n}\right)\right| \mathrm{d} x \mathrm{~d} t \\
= & I_{1, k n l}+I_{2, k n l}+I_{3, k n l}+I_{4, k n l}
\end{aligned}
$$

for some (small) constant $c_{p}>0$ of the uniformly monotonicity of the map $\mathrm{m} \mapsto$ $|\mathrm{m}|^{p-2} \mathrm{~m}$ (it needs $p \geq 2$, which indeed is implied by (4.4). From (4.13), from the strong convergence of $\theta_{k n l}$, and from the weak convergence of $\dot{\mathrm{m}}_{k n l}$ it follows that $I_{1, k n l} \rightarrow 0, I_{2, k n l} \rightarrow 0$ and $I_{3, k n l} \rightarrow 0$. From the identity $\mathrm{m}_{k n l} \times \dot{\mathrm{m}}_{k n l} \cdot \dot{\mathrm{m}}_{k n l}=0$ and the weak convergence of $\dot{\mathrm{m}}_{k n l}$ we obtain

$$
I_{4, k n l}=\int_{Q}\left|\frac{\mathrm{m}_{k n l}}{g\left(\left|\mathrm{~m}_{k n l}\right|\right)} \times \dot{\mathrm{m}}_{k n l} \cdot \dot{\mathrm{m}}_{k n}\right| \mathrm{d} x \mathrm{~d} t \rightarrow 0 .
$$

Altogether, the right-hand side of (4.16) indeed converges to zero, which proves the needed strong convergence $\dot{\mathrm{m}}_{k n l} \rightarrow \dot{\mathrm{m}}_{k n}$ even in $L^{p}\left(Q ; \mathbb{R}^{3}\right)$.

To show that $\mathrm{e}_{k n l} \rightarrow \mathrm{e}_{k n}$ in $L^{2}\left(Q ; \mathbb{R}^{3}\right)$, we use a similar argument: we test the approximated Maxwell system by $\mathrm{h}_{k n l}-\mathrm{h}_{k n}$ and $\mathrm{e}_{k n l}-\mathrm{e}_{k n}$, benefiting from the fact that we have $k$ fixed so that these test functions are admissible. Benefiting also from the smoothness of these test functions, we can use the curl-cancellation identity 
$\int_{\Omega} \operatorname{curl}\left(\mathrm{e}_{k n l}-\mathrm{e}_{k n}\right) \cdot\left(\mathrm{h}_{k n l}-\mathrm{h}_{k n}\right)-\operatorname{curl}\left(\mathrm{h}_{k n l}-\mathrm{h}_{k n}\right) \cdot\left(\mathbf{e}_{k n l}-\mathbf{e}_{k n}\right) \mathrm{d} x=0$. Then, proceeding as in (4.16), we show that

$$
\begin{aligned}
\lim _{l \rightarrow \infty} & \frac{\mu_{0}}{2}\left\|\mathbf{h}_{k n l}-\mathrm{h}_{k n}\right\|_{L^{\infty}\left(L^{2}\left(\Omega ; \mathbb{R}^{3}\right)\right)}^{2}+\int_{Q} \sigma\left(\theta_{k n}\right)\left|\mathbf{e}_{k n l}-\mathbf{e}_{k n}\right|^{2} \\
& +\frac{1}{n}\left(\left|\mathbf{e}_{k n l}\right|^{p-2} \mathbf{e}_{k n l}-\left|\mathbf{e}_{k n}\right|^{p-2} \mathbf{e}_{k n}\right) \cdot\left(\mathbf{e}_{k n l}-\mathbf{e}_{k n}\right) \mathrm{d} x \mathrm{~d} t=0,
\end{aligned}
$$

so that $\mathrm{e}_{k n l} \rightarrow \mathrm{e}_{k n}$ in $L^{2}\left(Q ; \mathbb{R}^{3}\right)$ is proved, and the limit passage in the heat equation is then easy.

Lemma 4.2. (Non-negativity of temperature) It holds $\theta_{k n} \geq 0$ and thus, in fact, $\theta_{k n}^{+}$ in (4.5) can be replaced by $\theta_{k n}$.

Proof. As standard, we exploit that $\theta_{k n}^{-}(t, \cdot) \in W^{1,2}(\Omega)$ and we test $(4.5 \mathrm{~b})$ by $\theta_{k n}^{-}$to show that $\theta_{k n}^{-}=0$. We show that all the right-hand side terms in (4.5b) either vanish or are negative under this test.

Lemma 4.3. (A priori estimates) There exists a constant $C$ not dependent on $k$ and $n$ such that

$$
\begin{aligned}
&\left\|\mathrm{m}_{k n}\right\|_{L^{\infty}\left(I ; W^{1,2}\left(\Omega ; \mathbb{R}^{3}\right)\right)} \leq C, \\
&\left\|\varphi_{0}\left(\mathrm{~m}_{k n}\right)\right\|_{L^{\infty}\left(I ; L^{1}(\Omega)\right)} \leq C, \leq \mathrm{h}_{k n} \|_{L^{\infty}\left(I ; L^{2}\left(\mathbb{R}^{3} ; \mathbb{R}^{3}\right)\right)} \leq C, \\
&\left\|\theta_{k n}\right\|_{L^{\infty}\left(I ; L^{\omega}(\Omega)\right)} \leq C,
\end{aligned}
$$

and also the estimates which are not uniform in $n$ :

$$
\begin{gathered}
\left\|\dot{\mathrm{m}}_{k n}\right\|_{L^{p}\left(Q ; \mathbb{R}^{3}\right)} \leq C n^{-1 / p}, \\
\left\|\mathrm{e}_{k n}\right\|_{L^{p}\left(Q ; \mathbb{R}^{3}\right)} \leq C n^{-1 / p} .
\end{gathered}
$$

Proof. It follows immediately by using $\dot{\mathrm{m}}_{k n}, 1, \mathrm{~h}_{k n}$ and $\mathrm{e}_{k n}$ as tests respectively for particular equations in (4.5). Integrating over a time interval $[0, t]$, summing it up, and using the curl-cancellation $\int_{\Omega} \operatorname{curl} \mathrm{e}_{k n} \cdot \mathrm{h}_{k n}-\operatorname{curl}_{k n} \cdot \mathrm{e}_{k n} \mathrm{~d} x=0$ in the Maxwell system itself, and also the cancellation of the adiabatic term $\theta_{k n}^{+} \varphi_{1}^{\prime}\left(\mathrm{m}_{k n}\right) \cdot \dot{\mathrm{m}}_{k n}$ (now written as $\theta_{k n} \varphi_{1}^{\prime}\left(\mathrm{m}_{k n}\right) \cdot \dot{\mathrm{m}}_{k n}$ in view of Lemma 4.2) between the magnetic and the heat equation, and further the cancellation of all the dissipative terms $\sigma\left(\theta_{k n}\right)\left|\mathbf{e}_{k n}\right|^{2}$, $\alpha\left|\dot{\mathrm{m}}_{k n}\right|^{2}$ and $\varrho_{n}^{\prime}\left(\dot{\mathrm{m}}_{k n}\right) \cdot \dot{\mathrm{m}}_{k n}$, we obtain the identity

$$
\begin{gathered}
\int_{\Omega} \frac{\mu_{0}}{2}\left|\mathrm{~h}_{k n}(t, \cdot)\right|^{2}+\varphi_{0}\left(\mathrm{~m}_{k n}(t, \cdot)\right)+\frac{\lambda}{2}\left|\nabla \mathrm{m}_{k n}(t, \cdot)\right|^{2}+\hat{c}\left(\theta_{k n}(t, \cdot)\right) \mathrm{d} x \\
+\frac{1}{n} \int_{0}^{t} \int_{\Omega}\left|\dot{\mathrm{m}}_{k n}\right|^{p}+\left|\mathrm{e}_{k n}\right|^{p} \mathrm{~d} x+\int_{0}^{t} \int_{\partial \Omega} \kappa_{\mathrm{b}} \theta_{k n} \mathrm{~d} S \mathrm{~d} t \\
=\int_{0}^{t}\left(\int_{\partial \Omega} \kappa_{\mathrm{b}} \theta_{\mathrm{e}} \mathrm{d} S-\int_{\Omega} \mu_{0} \mathrm{~h}_{\mathrm{e}} \cdot \dot{\mathrm{m}}_{k n} \mathrm{~d} x\right) \mathrm{d} t
\end{gathered}
$$




$$
\begin{aligned}
& +\int_{\Omega} \frac{\mu_{0}}{2}\left|\mathrm{~h}_{0, k}\right|^{2}+\varphi_{0}\left(\mathrm{~m}_{0, k}\right)+\frac{\lambda}{2}\left|\nabla \mathrm{m}_{0, k}\right|^{2}+\hat{c}\left(\theta_{0, k}\right) \mathrm{d} x \\
= & \int_{0}^{t}\left(\int_{\partial \Omega} \kappa_{\mathrm{b}} \theta_{\mathrm{e}} \mathrm{d} S+\int_{\Omega} \mu_{0} \dot{\mathrm{h}}_{\mathrm{e}} \cdot \mathrm{m}_{k n} \mathrm{~d} x\right) \mathrm{d} t+\int_{\Omega} \frac{\mu_{0}}{2}\left|\mathrm{~h}_{0, k}\right|^{2}+\varphi_{0}\left(\mathrm{~m}_{0, k}\right) \\
& +\frac{\lambda}{2}\left|\nabla \mathrm{m}_{0, k}\right|^{2}+\hat{c}\left(\theta_{0, k}\right)+\mu_{0} \mathrm{~h}_{\mathrm{e}}(0, \cdot) \cdot \mathrm{m}_{0, k}-\mu_{0} \mathrm{~h}_{\mathrm{e}}(t, \cdot) \cdot \mathrm{m}_{k n}(t, \cdot) \mathrm{d} x .
\end{aligned}
$$

Then we estimate the term $\left|\int_{\Omega} \mu_{0} \dot{\mathrm{h}}_{\mathrm{e}} \cdot \mathrm{m}_{k n} \mathrm{~d} x\right| \leq \mu_{0}\left\|\dot{\mathrm{h}}_{\mathrm{e}}\right\|_{L^{q^{\prime}}\left(\Omega ; \mathbb{R}^{3}\right)}\left\|\mathrm{m}_{k n}\right\|_{L^{q}\left(\Omega ; \mathbb{R}^{3}\right)}$ and treat it by the Gronwall inequality, relying on the coercivity of $\Phi: \mathrm{m} \mapsto \int_{\Omega} \varphi_{0}(\mathrm{~m})+$ $\frac{\lambda}{2}|\nabla \mathrm{m}|^{2} \mathrm{~d} x$ and the qualification of $\dot{\mathrm{h}}_{\mathrm{e}} \in L^{1}\left(I ; L^{2}\left(\Omega ; \mathbb{R}^{3}\right)\right)$, cf. $(3.2 \mathrm{k})$. The last term can be estimated as $\int_{\Omega} \mu_{0} \mathrm{~h}_{\mathrm{e}}(t, \cdot) \cdot \mathrm{m}_{k n}(t, \cdot) \mathrm{d} x \leq \mu_{0}\left\|\mathrm{~h}_{\mathrm{e}}(t, \cdot)\right\|_{L^{2}\left(\Omega ; \mathbb{R}^{3}\right)}\left\|\mathrm{m}_{k n}(t, \cdot)\right\|_{L^{2}\left(\Omega ; \mathbb{R}^{3}\right)}$ and then treated by Young's inequality, relying on the coercivity $\Phi$ and on $(3.2 \mathrm{k})$. Thus we obtain (4.19).

The next step is to derive a bound for $\nabla \theta_{k n}$.

Proposition 4.1. (Further estimates) There exists $1 \leq r<\frac{2 \omega+3}{\omega+3}$, and a constant $C>0$ such that

$$
\begin{aligned}
\left\|\nabla \theta_{k n}\right\|_{L^{r}\left(Q ; \mathbb{R}^{3}\right)} & \leq C, \\
\left\|\dot{\mathrm{m}}_{k n}\right\|_{L^{2}\left(Q ; \mathbb{R}^{3}\right)} & \leq C, \\
\left\|\mathrm{e}_{k n}\right\|_{L^{2}\left(Q ; \mathbb{R}^{3}\right)} & \leq C, \\
\left\|\dot{\theta}_{k n}\right\|_{L^{1}\left(I ;\left(W_{0}^{1, \infty}(\Omega)\right)^{*}\right)} & \leq C .
\end{aligned}
$$

Proof. For the reader's convenience, we organize the proof in successive steps.

Step 1. Nonlinear test of the heat equation. In the heat equation $(4.5 \mathrm{~b})$ we choose as test function

$$
\phi\left(\theta_{k n}\right):=1-\frac{1}{\left(1+\theta_{k n}\right) \zeta},
$$

where $\zeta>0$. Such a test yields the estimate (see Ref. 21 for details):

$$
\int_{Q} \phi^{\prime}\left(\theta_{k n}\right)\left|\nabla \theta_{k n}\right|^{2} \mathrm{~d} x \mathrm{~d} t \leq C\left(1+\left\|R_{k n}\right\|_{L^{1}(Q)}\right),
$$

where

$$
R_{k n}:=\alpha\left|\dot{\mathrm{m}}_{k n}\right|^{2}+\varrho_{n}^{\prime}\left(\dot{\mathrm{m}}_{k n}\right) \cdot \dot{\mathrm{m}}_{k n}+\sigma\left(\theta_{k n}\right)\left|\mathrm{e}_{k n}\right|^{2}+\theta_{k n} \varphi_{1}^{\prime}\left(\mathrm{m}_{k n}\right) \cdot \dot{\mathrm{m}}_{k n}
$$

Step 2. Interpolation. We show that for

$$
\zeta=\frac{2 \omega+3-r(\omega+3)}{3}
$$

the following estimate holds:

$$
\left\|\nabla \theta_{k n}\right\|_{L^{r}\left(Q ; \mathbb{R}^{3}\right)}^{r} \leq C \int_{Q} \phi^{\prime}\left(\theta_{k n}\right)\left|\nabla \theta_{k n}\right|^{2} \mathrm{~d} x \mathrm{~d} t .
$$


Note that the restriction $r<\frac{2 \omega+3}{\omega+3}$ guarantees that $\zeta$ from (4.24) is positive, as required in Step 1.

To begin with, we apply Hölder's inequality to obtain

$$
\begin{aligned}
\int_{Q}\left|\nabla \theta_{k n}\right|^{r} \mathrm{~d} x \mathrm{~d} t & =\int_{Q} \frac{\left|\phi^{\prime}\left(\theta_{k n}\right)\right|^{r / 2}\left|\nabla \theta_{k n}\right|^{r}}{\left|\phi^{\prime}\left(\theta_{k n}\right)\right|^{r / 2}} \mathrm{~d} x \mathrm{~d} t \\
& \leq\left(\int_{Q}\left|\phi^{\prime}\left(\theta_{k n}\right)\right|^{-\frac{r}{2-r}} \mathrm{~d} x \mathrm{~d} t\right)^{1-r / 2}\left(\int_{Q} \phi^{\prime}\left(\theta_{k n}\right)\left|\nabla \theta_{k n}\right|^{2} \mathrm{~d} x \mathrm{~d} t\right)^{r / 2} .
\end{aligned}
$$

Then, we observe that, since $\phi^{\prime}\left(\theta_{k n}\right)=\frac{\zeta}{(1+\theta k n)^{1+\zeta}}$,

$$
\int_{Q}\left|\phi^{\prime}\left(\theta_{k n}\right)\right|^{-\frac{r}{2-r}} \mathrm{~d} x \mathrm{~d} t=C_{\zeta, r}\left\|1+\theta_{k n}\right\|_{L^{p}(Q)}^{p}, \quad \text { where }\left\{\begin{array}{c}
C_{\zeta, r}=\zeta^{-\frac{r}{2-r}} \\
p=\frac{r(1+\zeta)}{2-r} .
\end{array}\right.
$$

Moreover, by $(4.24)$ we have $\zeta=(2-r)(\omega / 3+1)-1$, and thus from (4.27) we obtain $p / r=\omega / 3+1$, that is to say,

$$
-\frac{\lambda}{3}+\frac{1-\lambda}{\omega}=0, \quad \text { where } \lambda=\frac{r}{p} \in(0,1),
$$

whence

$$
\frac{1}{p}=\frac{\lambda}{r}=\lambda\left(\frac{1}{r}-\frac{1}{3}\right)+\frac{1-\lambda}{\omega} .
$$

Furthermore, by (4.28) and by Gagliardo-Nirenberg's interpolation inequality between $L^{\omega}(\Omega)$ and $W^{1, r}(\Omega)$, we have

$$
\left\|1+\theta_{k n}(t, \cdot)\right\|_{L^{p}(\Omega)}^{p} \leq C\left\|1+\theta_{k n}(t, \cdot)\right\|_{L^{\omega}(\Omega)}^{p(1-\lambda)}\left\|1+\theta_{k n}(t, \cdot)\right\|_{W^{1, r}(\Omega)}^{r}
$$

for a.e. $t \in(0, T)$. From (4.29) and (4.19d) we obtain

$$
\left\|1+\theta_{k n}\right\|_{L^{p}(Q)}^{p} \leq C\left(1+\int_{Q}\left|\nabla \theta_{k n}\right|^{r} \mathrm{~d} x \mathrm{~d} t\right) .
$$

Finally, on combining (4.26), (4.27) and (4.30) we obtain (4.25).

Step 3. Estimation of the right-hand side $R_{k n}$. We prove the following estimate:

$$
\left\|R_{k n}\right\|_{L^{1}(Q)} \leq C\left(1+\int_{0}^{T}\left\|\theta_{k n}\right\|_{L^{s}(\Omega)}^{2} \mathrm{~d} t\right), \quad s:=\frac{2 q_{1}}{q_{1}-2},
$$

with $q_{1}$ as in $(3.2 \mathrm{j})$.

To show that (4.31) holds, let us abbreviate

$$
\begin{aligned}
I_{k n} & :=\int_{Q} \alpha\left|\dot{\mathrm{m}}_{k n}\right|^{2}+\varrho_{n}^{\prime}\left(\dot{\mathrm{m}}_{k n}\right) \cdot \dot{\mathrm{m}}_{k n}+\sigma\left(\theta_{k n}\right)\left|\mathrm{e}_{k n}\right|^{2} \mathrm{~d} x \mathrm{~d} t, \\
J_{k n} & :=\int_{Q}\left|\theta_{k n} \varphi_{1}^{\prime}\left(\mathrm{m}_{k n}\right) \cdot \dot{\mathrm{m}}_{k n}\right| \mathrm{d} x \mathrm{~d} t .
\end{aligned}
$$


Using Hölder's inequality, (3.2j) and (4.19b), and Young's inequality, we obtain

$$
\begin{aligned}
J_{k n} & \leq \int_{0}^{T}\left\|\varphi_{1}^{\prime}\left(\mathrm{m}_{k n}\right)\right\|_{L^{q_{1}(\Omega)}}\left\|\theta_{k n}\right\|_{L^{s}(\Omega)}\left\|\dot{\mathrm{m}}_{k n}\right\|_{L^{2}(\Omega)} \mathrm{d} t \\
& \leq C \int_{0}^{T}\left\|\theta_{k n}\right\|_{L^{s}(\Omega)}\left\|\dot{\mathrm{m}}_{k n}\right\|_{L^{2}(\Omega)} \mathrm{d} t \\
& \leq C_{\delta} \int_{0}^{T}\left\|\theta_{k n}\right\|_{L^{s}(\Omega)}^{2} \mathrm{~d} t+\delta I_{k n},
\end{aligned}
$$

where $\delta>0$ can be arbitrarily small and $C_{\delta}>0$ depends on $\delta$. Testing the discrete Gilbert's equation and the two discrete Maxwell's equations (4.5a), (4.5c) and (4.5d) respectively, by $\dot{\mathrm{m}}_{k n}, \mathrm{~h}_{k n}$ and $\mathrm{e}_{k n}$, and summing them we obtain, by curl cancellation,

$$
\begin{aligned}
\int_{\Omega} \frac{\mu_{0}}{2}\left|\mathrm{~h}_{k n}(T, \cdot)\right|^{2}+\varphi_{0}\left(\mathrm{~m}_{k n}(T, \cdot)\right)+\frac{\lambda}{2}\left|\nabla \mathrm{m}_{k n}(T, \cdot)\right|^{2} \mathrm{~d} x \\
\quad+\frac{1}{n} \int_{Q}\left|\dot{\mathrm{m}}_{k n}\right|^{p}+\left|\mathrm{e}_{k n}\right|^{p}+\alpha\left|\dot{\mathrm{m}}_{k n}\right|^{2}+\varrho_{n}^{\prime}\left(\dot{\mathrm{m}}_{k n}\right) \cdot \dot{\mathrm{m}}_{k n}+\sigma\left(\theta_{k n}\right)\left|\mathrm{e}_{k n}\right|^{2} \mathrm{~d} x \mathrm{~d} t \\
=\int_{\Omega} \frac{\mu_{0}}{2}\left|\mathrm{~h}_{0, k}\right|^{2}+\varphi_{0}\left(\mathrm{~m}_{0, k}\right)+\frac{\lambda}{2}\left|\nabla \mathrm{m}_{0, k}\right|^{2}+\hat{c}\left(\theta_{0, k}\right) \mathrm{d} x \\
\quad+\int_{Q} \theta_{k n} \varphi_{1}^{\prime}\left(\mathrm{m}_{k n}\right) \dot{\mathrm{m}}_{k n} \mathrm{~d} x \mathrm{~d} t+\int_{Q} \mu_{0} \mathrm{~h}_{\mathrm{e}} \cdot \dot{\mathrm{m}}_{k n} \mathrm{~d} x \mathrm{~d} t .
\end{aligned}
$$

In particular, we have

$$
I_{k n} \leq C+J_{k n}+\int_{Q} \mu_{0} \mathrm{~h}_{\mathrm{e}} \cdot \dot{\mathrm{m}}_{k n} \mathrm{~d} x \mathrm{~d} t .
$$

We can treat the term $\int_{Q} \mu_{0} \mathrm{~h}_{\mathrm{e}} \cdot \dot{\mathrm{m}}_{k n} \mathrm{~d} x \mathrm{~d} t$ by Hölder's and Young's inequalities, and then by absorption of $\dot{m}$ on the left-hand side, we obtain

$$
I_{k n} \leq C\left(1+J_{k n}\right) .
$$

Taking $\delta$ small enough in (14) and using (4.34), we eventually arrive at (4.31).

Step 4. Further interpolation. (This step is to be skipped if $\omega \geq 3$.) We prove the following: if $\omega<3$ and the exponent $s$ defined in (4.31) satisfies $s<\frac{6 \omega}{\omega+3}$, then there exist $r \in\left[1, \frac{2 \omega+3}{\omega+3}\right)$ and $\lambda \in(0, r / 2)$ such that

$$
\int_{0}^{T}\left\|\theta_{k n}\right\|_{L^{s}(\Omega)}^{2} \mathrm{~d} t \leq C\left(1+\left\|\nabla \theta_{k n}\right\|_{L^{r}\left(Q ; \mathbb{R}^{3}\right)}^{r}\right)^{\frac{2 \lambda}{r}} .
$$

By direct computation we verify that if $\omega<3$, then

$$
\inf _{\substack{1 \leq r<\frac{2 \omega+3}{\omega+3} \\ 0<\lambda<r / 2}} \frac{1-\lambda}{\omega}+\lambda\left(\frac{1}{r}-\frac{1}{3}\right)=\frac{\omega+3}{6 \omega} .
$$


Hence we can select $r \in\left[1, \frac{2 \omega+3}{\omega+3}\right)$ and $\lambda \in(0, r / 2)$ such that

$$
\frac{1}{s} \geq \frac{1-\lambda}{\omega}+\lambda\left(\frac{1}{r}-\frac{1}{3}\right)
$$

By (4.36) we can interpolate between $L^{\omega}(\Omega)$ and $W^{1, r}(\Omega)$ and we can use the bound in $L^{\infty}\left((0, T) ; L^{\omega}(\Omega)\right)$ for $\theta_{k n}$ (see $\left.(4.19 \mathrm{~d})\right)$ to obtain, for a.e. $t \in(0, T)$,

$$
\begin{aligned}
\left\|\theta_{k n}(t, \cdot)\right\|_{L^{s}(\Omega)} & \leq C_{1}\left\|\theta_{k n}(t, \cdot)\right\|_{L^{\omega}(\Omega)}^{1-\lambda}\left\|\theta_{k n}(t, \cdot)\right\|_{W^{1, r}(\Omega)}^{\lambda} \\
& \leq C_{2}\left(1+\left\|\nabla \theta_{k n}(t, \cdot)\right\|_{L^{r}\left(\Omega ; \mathbb{R}^{3}\right)}^{\lambda}\right) .
\end{aligned}
$$

Thus,

$$
\int_{0}^{T}\left\|\theta_{k n}\right\|_{L^{s}(\Omega)}^{2} \mathrm{~d} t \leq C\left(1+\int_{0}^{T}\left\|\nabla \theta_{k n}\right\|_{L^{r}\left(\Omega ; \mathbb{R}^{3}\right)}^{2 \lambda} \mathrm{d} t\right) .
$$

Since $r /(2 \lambda)>1$, we now use Hölder's inequality to obtain the bound (4.35) from (4.38).

Step 5. "Isotropic" estimate for temperature. We collect the inequalities (4.23), (4.25) and (4.31) to obtain

$$
\left\|\theta_{k n}\right\|_{L^{r}\left(Q ; \mathbb{R}^{3}\right)}^{r} \leq C_{1}\left(1+\left\|R_{k n}\right\|_{L^{1}(Q)}\right) \leq C_{2}\left(1+\int_{0}^{T}\left\|\theta_{k n}\right\|_{L^{s}(\Omega)}^{2} \mathrm{~d} t\right),
$$

and we prove the estimate (4.21a) by showing that

$$
\int_{0}^{T}\left\|\theta_{k n}\right\|_{L^{s}(\Omega)}^{2} \mathrm{~d} t \leq C .
$$

To prove (4.40), we consider two cases, according to whether $\omega<3$ or $\omega \geq 3$.

(a) Case $\omega<3$. In this case $\frac{2 \omega}{\omega-2}>\frac{6 \omega}{2 \omega-3}$, thus by $(3.2 \mathrm{j})$ and $(4.31)$ we have that $s<\frac{6 \omega}{\omega+3}$ and hence we can use the result proved in Step 4. By (4.35) and (4.39) we have

$$
\left(\int_{0}^{T}\left\|\theta_{k n}\right\|_{L^{s}(\Omega)} \mathrm{d} t\right)^{\frac{r}{2 \lambda}} \leq C\left(1+\int_{0}^{T}\left\|\theta_{k n}\right\|_{L^{s}(\Omega)} \mathrm{d} t\right) .
$$

Now recall from Step 4 that $\lambda \in(0, r / 2)$, hence (4.41) implies (4.40).

(b) Case $\omega \geq 3$. In this case $\frac{2 \omega}{\omega-2} \leq \frac{6 \omega}{2 \omega-3}$, thus (3.2j) implies $\frac{2 q_{1}}{q_{1}-2} \leq \omega$, and therefore $s \leq \omega$ by (4.31). Hence, from (4.19d) we obtain again the estimate (4.40).

Step 6. Boundedness of heat sources. We now prove (4.21b) and (4.21c). From (4.39) and (4.40) we have

$$
\left\|R_{k n}\right\|_{L^{1}(Q)} \leq C
$$

Moreover, by the linear growth of $\varrho(\cdot)$, by $(4.2)$, and by the assumption that $\sigma(\cdot)$ is bounded from below (cf. (3.2f)) it follows that

$$
\left\|\dot{\mathrm{m}}_{k n}\right\|_{L^{2}\left(Q ; \mathbb{R}^{3}\right)}+\left\|\mathrm{e}_{k n}\right\|_{L^{2}\left(Q ; \mathbb{R}^{3}\right)} \leq C\left\|R_{k n}\right\|_{L^{1}(Q)} .
$$

The estimates (4.21b) and (4.21c) follow immediately from (4.42) and (4.43). 
Step 7. Time-derivative of temperature. In this final step we prove the estimate $(4.21 \mathrm{~d})$. Let $w \in L^{\infty}\left(I ; W_{0}^{1, \infty}(\Omega)\right)$. By $(3.2 \mathrm{~b})$ and $(3.2 \mathrm{~d})$ we can take $z=w / c\left(\theta_{k n}\right)$ as test function in (4.5b) to obtain:

$$
\begin{aligned}
\int_{Q} \dot{\theta}_{k n} w \mathrm{~d} x \mathrm{~d} t= & \int_{Q} \frac{R_{k n} w}{c\left(\theta_{k n}\right)}-\frac{\kappa\left(\theta_{k n}\right) \nabla \theta_{k n} \cdot \nabla w}{c\left(\theta_{k n}\right)} \\
& +\frac{c^{\prime}\left(\theta_{k n}\right) \kappa\left(\theta_{k n}\right) \nabla \theta_{k n} \cdot \nabla \theta_{k n} w}{c\left(\theta_{k n}\right)^{2}} \mathrm{~d} x \mathrm{~d} t .
\end{aligned}
$$

By $(3.2 \mathrm{~d}),(4.42)$ and $(4.21 \mathrm{a})$ we have

$$
\int_{Q} \frac{R_{k n} w}{c\left(\theta_{k n}\right)}-\frac{\kappa\left(\theta_{k n}\right) \nabla \theta_{k n} \cdot \nabla w}{c\left(\theta_{k n}\right)} \mathrm{d} x \mathrm{~d} t \leq C\|w\|_{L^{\infty}\left(I ; W_{0}^{1, \infty}(\Omega)\right)} ;
$$

moreover, by $(3.2 \mathrm{~g}),(4.22)$ and $(4.23)$,

$$
\begin{aligned}
\int_{Q} \frac{c^{\prime}\left(\theta_{k n}\right) \kappa\left(\theta_{k n}\right) \nabla \theta_{k n} \cdot \nabla \theta_{k n} w}{c\left(\theta_{k n}\right)^{2}} \mathrm{~d} x \mathrm{~d} t & \leq C_{1} \int_{Q} \frac{\phi^{\prime}\left(\theta_{k n}\right)\left|\nabla \theta_{k n}\right|^{2} w}{c\left(\theta_{k n}\right)^{2}} \mathrm{~d} x \mathrm{~d} t \\
& \leq C_{2}\|w\|_{L^{\infty}(Q)} .
\end{aligned}
$$

From $(4.44)-(4.46)$ we conclude that $\sup \left\{\int_{Q} \dot{\theta}_{k n} w \mathrm{~d} x \mathrm{~d} t:\|w\|_{L^{\infty}\left(I ; W_{0}^{1, \infty}(\Omega)\right)}=1\right\} \leq C$, whence $(4.21 \mathrm{~d})$.

Lemma 4.4. (Passage $n \rightarrow \infty)$ A selected subsequence $\left\{\left(\mathrm{m}_{k n}, \theta_{k n}, \mathrm{~h}_{k n}, \mathrm{e}_{k n}\right)\right\}_{n \in \mathbb{N}}$ does exist such that it converges weakly* in the topologies indicated by $(4.19 \mathrm{a})-(4.19 \mathrm{e})$ and (4.21) to a limit $\left(\mathrm{m}_{k}, \theta_{k}, \mathrm{e}_{k}, \mathrm{~h}_{k}\right)$ satisfying the initial conditions (4.6) such that $\dot{\mathrm{h}}_{k} \in L^{2}\left(I ;\left(V_{k}\right)^{*}\right)$. Moreover, there exists $\mathrm{r}_{k} \in L^{2}\left(Q ; \mathbb{R}^{3}\right)$ such that

(i) for a.a. $t \in I$, for all $\mathrm{z} \in V_{k}^{3}$ and all $\mathrm{w} \in L^{2}\left(\Omega ; \mathbb{R}^{3}\right)$

$$
\begin{gathered}
\int_{\Omega}\left(\mathrm{r}_{k}+\varphi_{0}^{\prime}\left(\mathrm{m}_{k}\right)-\frac{\mathrm{m}_{k} \times \dot{\mathrm{m}}_{k}}{g\left(\left|\mathrm{~m}_{k}\right|\right)}+\theta_{k} \varphi_{1}^{\prime}\left(\mathrm{m}_{k}\right)-\mu_{0} \mathrm{~h}_{k}-\mu_{0} \mathrm{~h}_{\mathrm{e}}\right) \cdot \mathbf{z} \\
+\lambda \nabla \mathrm{m}_{k}: \nabla \mathbf{z} \mathrm{d} x=0, \\
\int_{\Omega} \varrho(\mathbf{w})-\varrho\left(\dot{\mathrm{m}}_{k}\right)-\left(\mathbf{r}_{k}-\alpha \dot{\mathrm{m}}_{k}\right) \cdot\left(\mathbf{w}-\dot{\mathrm{m}}_{k}\right) \mathrm{d} x \geq 0 ;
\end{gathered}
$$

(ii) for all $z \in C^{1}(\bar{Q})$

$$
\begin{aligned}
& -\int_{Q} \hat{c}\left(\theta_{k}\right) \dot{\mathbf{z}}+\kappa\left(\theta_{k}\right) \nabla \theta_{k} \cdot \nabla z \mathrm{~d} x \mathrm{~d} t \\
& =\int_{Q}\left(\sigma\left(\theta_{k}\right)\left|\mathrm{e}_{k}\right|^{2}+\alpha\left|\dot{\mathrm{m}}_{k}\right|^{2}+\varrho\left(\dot{\mathrm{m}}_{k}\right)\right) z+\theta_{k} \varphi_{1}^{\prime}\left(\mathrm{m}_{k}\right) \cdot \dot{\mathrm{m}}_{k} z \mathrm{~d} x \mathrm{~d} t \\
& \quad+\int_{\Sigma} \kappa_{\mathrm{b}}\left(\theta_{\mathrm{e}, k}-\theta_{k}\right) z \mathrm{~d} S \mathrm{~d} t+\int_{\Omega} \hat{c}\left(\theta_{\mathrm{e}, k}\right) z(0, \cdot) \mathrm{d} x
\end{aligned}
$$


(iii) for a.a. $t \in I$, for all $\mathrm{u} \in U_{k}$ and $\mathrm{v} \in V_{k}^{3}$

$$
\begin{aligned}
& \int_{\Omega} \mu_{0} \dot{\mathrm{h}}_{k} \cdot \mathrm{u}+\mathrm{e}_{k} \cdot \operatorname{curl} \mathrm{u}+\mu_{0} \dot{\mathrm{m}}_{k} \cdot \mathrm{ud} x=0, \\
& \int_{\Omega} \mathrm{h}_{k} \cdot \operatorname{curl} \mathrm{v}-\sigma\left(\theta_{k}\right) \mathrm{e}_{k} \cdot \mathrm{vd} x=0 .
\end{aligned}
$$

Sketch of the Proof. The passage to the limit from (4.5) to (4.47) is the same as we did in the proof of Lemma 4.1, since $k$ is still constant. One difference is that we first introduce $\mathrm{r}_{k n}:=\alpha \dot{\mathrm{m}}_{k n}+\varrho_{n}^{\prime}\left(\dot{\mathrm{m}}_{k n}\right) \in L^{2}\left(Q ; \mathbb{R}^{3}\right)$. The a priori estimate $(4.21 \mathrm{~b})$ yields boundedness of $\left\{\mathbf{r}_{k n}\right\}_{n \in \mathbb{N}}$ so that we can also rely on $\mathbf{r}_{k n} \rightarrow \mathrm{r}_{k}$ weakly in $L^{2}\left(Q ; \mathbb{R}^{3}\right)$ for a selected subsequence. Realizing convexity of $\varrho_{n}$, we can thus rewrite (4.5a) into

$$
\begin{array}{r}
\int_{\Omega}\left(\mathrm{r}_{k n}+\varphi_{0}^{\prime}\left(\mathrm{m}_{k n}\right)-\frac{\mathrm{m}_{k n} \times \dot{\mathrm{m}}_{k n}}{g\left(\left|\mathrm{~m}_{k n}\right|\right)}+\theta_{k n} \varphi_{1}^{\prime}\left(\mathrm{m}_{k n}\right)-\mu_{0} \mathrm{~h}_{k n}\right. \\
\left.\quad-\mu_{0} \mathrm{~h}_{\mathrm{e}}+\frac{1}{n}\left|\dot{\mathrm{m}}_{k n}\right|^{p-2} \dot{\mathrm{m}}_{k n}\right) \cdot \mathbf{z}+\lambda \nabla \mathrm{m}_{k n}: \nabla \mathbf{z d} x=0, \\
\int_{\Omega} \varrho_{n}(\mathbf{w})-\varrho_{n}\left(\dot{\mathrm{m}}_{k n}\right)-\left(\mathrm{r}_{k n}-\alpha \dot{\mathrm{m}}_{k n}\right) \cdot\left(\mathbf{w}-\dot{\mathrm{m}}_{k n}\right) \mathrm{d} x \geq 0 .
\end{array}
$$

The estimate $(4.19 \mathrm{e})$ is now used to show that the regularizing term in the magnetic part vanishes in the limit. Indeed, we have

$$
\begin{aligned}
\left|\int_{Q} \frac{1}{n}\right| \dot{\mathrm{m}}_{k n}\left|{ }^{p-2} \dot{\mathrm{m}}_{k n} \cdot \mathbf{z d} x \mathrm{~d} t\right| & \leq \frac{1}{n}\left\|\dot{\mathrm{m}}_{k n}\right\|_{L^{p}\left(Q ; \mathbb{R}^{3}\right)}^{p-1}\|\mathbf{z}\|_{L^{p}\left(Q ; \mathbb{R}^{3}\right)} \\
& \leq C \frac{1}{\sqrt[p]{n}}\|\mathbf{z}\|_{L^{p}\left(Q ; \mathbb{R}^{3}\right)} \rightarrow 0
\end{aligned}
$$

for any $\mathbf{z} \in L^{p}\left(Q ; \mathbb{R}^{3}\right)$. This allows us to pass from (4.48a) to (4.47a) if integrated over time because the other terms can be converged easily.

Now we want to estimate limit superior in (4.48b) if integrated over time. Using (4.2c) and (4.2a) we get

$$
\begin{aligned}
\liminf _{n \rightarrow \infty} \int_{Q} \varrho_{n}\left(\dot{\mathrm{m}}_{k n}\right) \mathrm{d} x \mathrm{~d} t= & \lim _{n \rightarrow \infty} \int_{Q} \varrho_{n}\left(\dot{\mathrm{m}}_{k n}\right)-\varrho\left(\dot{\mathrm{m}}_{k n}\right) \mathrm{d} x \mathrm{~d} t \\
& +\liminf _{n \rightarrow \infty} \int_{Q} \varrho\left(\dot{\mathrm{m}}_{k n}\right) \mathrm{d} x \mathrm{~d} t \geq \int_{Q} \varrho\left(\dot{\mathrm{m}}_{k}\right) \mathrm{d} x \mathrm{~d} t .
\end{aligned}
$$

The only difficult term $\mathbf{r}_{k n} \cdot \dot{\mathrm{m}}_{k n}$ remains to be treated by using (4.48a) tested by $\mathbf{z}=\dot{\mathrm{m}}_{k n}$ and further using the already proved limit in (4.48a), namely

$$
\begin{aligned}
\limsup _{n \rightarrow \infty} & \int_{Q} \mathrm{r}_{k n} \cdot \dot{\mathrm{m}}_{k n} \mathrm{~d} x \mathrm{~d} t \\
= & \limsup _{n \rightarrow \infty} \int_{Q}\left(\frac{\mathrm{m}_{k n} \times \dot{\mathrm{m}}_{k n}}{g\left(\left|\mathrm{~m}_{k n}\right|\right)}-\varphi_{0}^{\prime}\left(\mathrm{m}_{k n}\right)-\theta_{k n} \varphi_{1}^{\prime}\left(\mathrm{m}_{k n}\right)\right. \\
& \left.\quad+\mu_{0} \mathrm{~h}_{k n}+\mu_{0} \mathrm{~h}_{\mathrm{e}}-\frac{1}{n}\left|\dot{\mathrm{m}}_{k n}\right|^{p-2} \dot{\mathrm{m}}_{k n}\right) \cdot \dot{\mathrm{m}}_{k n}-\lambda \nabla \mathrm{m}_{k n}: \nabla \dot{\mathrm{m}}_{k n} \mathrm{~d} x \mathrm{~d} t
\end{aligned}
$$




$$
\begin{aligned}
\leq & \lim _{n \rightarrow \infty} \int_{Q}\left(\mu_{0} \mathrm{~h}_{k n}+\mu_{0} \mathrm{~h}_{\mathrm{e}}-\varphi_{0}^{\prime}\left(\mathrm{m}_{k n}\right)-\theta_{k n} \varphi_{1}^{\prime}\left(\mathrm{m}_{k n}\right)\right) \\
& \cdot \dot{\mathrm{m}}_{k n}-\lambda \nabla \mathrm{m}_{k n}: \nabla \dot{\mathrm{m}}_{k n} \mathrm{~d} x \mathrm{~d} t \\
= & \int_{Q}\left(\mu_{0} \mathrm{~h}_{k}+\mu_{0} \mathrm{~h}_{\mathrm{e}}-\varphi_{0}^{\prime}\left(\mathrm{m}_{k}\right)-\theta_{k} \varphi_{1}^{\prime}\left(\mathrm{m}_{k}\right)\right) \cdot \dot{\mathrm{m}}_{k}-\lambda \nabla \mathrm{m}_{k}: \nabla \dot{\mathrm{m}}_{k} \mathrm{~d} x \mathrm{~d} t \\
= & \int_{Q}\left(\frac{\mathrm{m}_{k} \times \dot{\mathrm{m}}_{k}}{g\left(\left|\mathrm{~m}_{k}\right|\right)}+\mu_{0} \mathrm{~h}_{k}+\mu_{0} \mathrm{~h}_{\mathrm{e}}-\varphi_{0}^{\prime}\left(\mathrm{m}_{k}\right)-\theta_{k} \varphi_{1}^{\prime}\left(\mathrm{m}_{k}\right)\right) \\
& \cdot \dot{\mathrm{m}}_{k}-\lambda \nabla \mathrm{m}_{k}: \nabla \dot{\mathrm{m}}_{k} \mathrm{~d} x \mathrm{~d} t \\
= & \int_{Q} \mathrm{r}_{k} \cdot \dot{\mathrm{m}}_{k} \mathrm{~d} x \mathrm{~d} t
\end{aligned}
$$

where we benefited from having the finite-dimensional approximation of $\mathrm{m}_{k n}(t, \cdot) \in V_{k}$. Thus the limit passage from (4.48b) to (4.47b) if integrated over time is done.

For limit passage in the heat equation, we need to improve $\dot{\mathrm{m}}_{k n} \rightarrow \dot{\mathrm{m}}_{k}$ strongly in $L^{2}\left(Q ; \mathbb{R}^{3}\right)$. To this goal, we modify the procedure (4.16) and (4.17) by omitting the $L^{p}$-term in (4.16) and, since now $\varrho_{n}$ is not fixed, by handling the $\varrho$-term as follows: for any $\xi_{k} \in L^{2}\left(Q ; \mathbb{R}^{3}\right)$ such that $\xi_{k} \in \partial \varrho\left(\dot{\mathrm{m}}_{k}\right)$ a.e. on $Q$, we have

$$
\begin{aligned}
\liminf _{n \rightarrow \infty} \int_{Q}\left(\varrho_{n}^{\prime}\left(\dot{\mathrm{m}}_{k n}\right)-\xi_{k}\right) \cdot\left(\dot{\mathrm{m}}_{k n}-\dot{\mathrm{m}}_{k}\right) \mathrm{d} x \mathrm{~d} t \\
=\liminf _{n \rightarrow \infty} \int_{Q}\left(\varrho_{n}^{\prime}\left(\dot{\mathrm{m}}_{k n}\right)-\varrho_{n}^{\prime}\left(\dot{\mathrm{m}}_{k}\right)\right) \cdot\left(\dot{\mathrm{m}}_{k n}-\dot{\mathrm{m}}_{k}\right) \\
\quad+\left(\varrho_{n}^{\prime}\left(\dot{\mathrm{m}}_{k}\right)-\xi_{k}\right) \cdot\left(\dot{\mathrm{m}}_{k n}-\dot{\mathrm{m}}_{k}\right) \mathrm{d} x \mathrm{~d} t \\
\geq \liminf _{n \rightarrow \infty} \int_{Q}\left(\varrho_{n}^{\prime}\left(\dot{\mathrm{m}}_{k}\right)-\xi_{k}\right) \cdot\left(\dot{\mathrm{m}}_{k n}-\dot{\mathrm{m}}_{k}\right) \mathrm{d} x \mathrm{~d} t \\
=\liminf _{n \rightarrow \infty} \int_{\left\{(t, x) \in Q ; \dot{\mathrm{m}}_{k}(t, x) \neq 0\right\}}\left(\varrho_{n}^{\prime}\left(\dot{\mathrm{m}}_{k}\right)-\xi_{k}\right) \cdot\left(\dot{\mathrm{m}}_{k n}-\dot{\mathrm{m}}_{k}\right) \mathrm{d} x \mathrm{~d} t \\
\quad+\lim _{n \rightarrow \infty} \int_{\left\{(t, x) \in Q ; \dot{\mathrm{m}}_{k}(t, x)=0\right\}}\left(\varrho_{n}^{\prime}\left(\dot{\mathrm{m}}_{k}\right)-\xi_{k}\right) \cdot\left(\dot{\mathrm{m}}_{k n}-\dot{\mathrm{m}}_{k}\right) \mathrm{d} x \mathrm{~d} t
\end{aligned}
$$

where we also used that $\varrho_{n}^{\prime}$ is monotone since $\varrho_{n}$ is convex, cf. (4.2a).

Due to $(4.2 \mathrm{~d})$, we have $\varrho_{n}^{\prime}\left(\dot{\mathrm{m}}_{k}\right)-\xi_{k} \rightarrow 0$ pointwise on $\left\{(t, x) \in Q ; \dot{\mathrm{m}}_{k}(t, x) \neq 0\right\}$ since $\xi_{k}=\varrho^{\prime}\left(\dot{\mathrm{m}}_{k}\right)$ on this set. Then the functions $\left|\varrho_{n}^{\prime}\left(\dot{\mathrm{m}}_{k}\right)-\xi_{k}\right|^{2}$, being bounded due to $(2.15)$ and $(4.2 \mathrm{a})$ on $Q$, have certainly a common integrable majorant and, by Lebesgue theorem, $\varrho_{n}^{\prime}\left(\dot{\mathrm{m}}_{k}\right)-\xi_{k} \rightarrow 0$ in $L^{2}(Q)$. The last but one term in (4.52) thus converges to 0 . The last term in (4.52) simply equals to $-\int_{\left\{(t, x) \in Q ; \dot{\mathrm{m}}_{k}(t, x)=0\right\}} \xi_{k}$. $\dot{\mathrm{m}}_{k n} \mathrm{~d} x \mathrm{~d} t$ due to $(4.2 \mathrm{~b})$ and, as such, it obviously converges to 0 for $n \rightarrow \infty$, too. Hence, the first term in (4.52) can be forgotten for the procedure (4.16) and (4.17), which then again shows that $\dot{\mathrm{m}}_{k n} \rightarrow \dot{\mathrm{m}}_{k}$ in $L^{2}\left(Q ; \mathbb{R}^{3}\right)$. 
Like before, we can execute the procedure like (4.18) which, however, now must be modified by replacing the term $\frac{1}{n}\left(\left|\mathbf{e}_{k n l}\right|^{p-2} \mathbf{e}_{k n l}-\left|\mathbf{e}_{k n}\right|^{p-2} \mathbf{e}_{k n}\right) \cdot\left(\mathbf{e}_{k n l}-\mathbf{e}_{k n}\right)$ by $\frac{1}{n}\left(\left|\mathbf{e}_{k n}\right|^{p-2} \mathbf{e}_{k n}\right) \cdot\left(\mathbf{e}_{k n}-\mathbf{e}_{k}\right)$, which can further be treated as

$$
\begin{gathered}
\liminf _{n \rightarrow \infty} \int_{Q} \frac{1}{n}\left(\left|\mathbf{e}_{k n}\right|^{p-2} \mathbf{e}_{k n}\right) \cdot\left(\mathbf{e}_{k n}-\mathbf{e}_{k}\right) \mathrm{d} x \mathrm{~d} t \\
\leq \lim _{n \rightarrow \infty} \int_{Q} \frac{1}{n}\left(\left|\mathbf{e}_{k n}\right|^{p-2} \mathbf{e}_{k n}\right) \cdot \mathbf{e}_{k} \mathrm{~d} x \mathrm{~d} t=0,
\end{gathered}
$$

where we also used (4.19f) to get

$$
\begin{aligned}
\left.\left|\int_{Q} \frac{1}{n}\right| \mathrm{e}_{k n}\right|^{p-2} \mathrm{e}_{k n} \cdot \mathrm{vd} x \mathrm{~d} t \mid & \leq \frac{1}{n}\left\|\mathrm{e}_{k n}\right\|_{L^{p}\left(Q ; \mathbb{R}^{3}\right)}^{p-1}\|\mathrm{v}\|_{L^{p}\left(Q ; \mathbb{R}^{3}\right)} \\
& \leq C \frac{1}{\sqrt[p]{n}}\|\mathrm{v}\|_{L^{p}\left(Q ; \mathbb{R}^{3}\right)} \rightarrow 0,
\end{aligned}
$$

to get for any $\mathbf{v} \in L^{p}\left(Q ; \mathbb{R}^{3}\right)$ and in particular for $\mathbf{v}=\mathrm{e}_{k}$; here it is important that $\mathbf{e}_{k}$ is not only in $L^{2}\left(Q ; \mathbb{R}^{3}\right)$ as seen from $(4.21 \mathrm{c})$ but indeed even in $L^{p}\left(Q ; \mathbb{R}^{3}\right)$. This last fact follows from that $\mathrm{e}_{k}(t, \cdot)$ ranges the finite-dimensional space $V_{k}^{3}$ and, by $(4.47 \mathrm{e})$, $\sigma\left(\theta_{k}\right) \mathrm{e}_{k} \in L^{\infty}\left(I ;\left(V_{k}^{3}\right)^{*}\right)$ so that

$$
\begin{aligned}
\int_{Q}\left|\mathrm{e}_{k}\right|^{4} \mathrm{~d} x \mathrm{~d} t & \leq C_{1} \int_{Q}\left(\sigma\left(\theta_{k}\right)\left|\mathbf{e}_{k}\right|^{2}\right)^{2} \mathrm{~d} x \mathrm{~d} t \leq C_{2} \int_{0}^{T}\left\langle\sigma\left(\theta_{k}\right) \mathbf{e}_{k}, \mathrm{e}_{k}\right\rangle_{V_{k}^{3}}^{2} \mathrm{~d} t \\
& \leq C_{3} \int_{0}^{T}\left\|\mathrm{e}_{k}\right\|_{V_{k}^{3}}^{2} \mathrm{~d} t \leq C_{4} \int_{Q}\left|\mathbf{e}_{k}\right|^{2} \mathrm{~d} x \mathrm{~d} t,
\end{aligned}
$$

where we have used the fact that $\sigma\left(\theta_{k}\right) \geq \sigma_{\min }>0$. Since $\mathrm{e}_{k} \in L^{4}\left(I ; V_{k}^{3}\right)$, we have that $\sigma\left(\theta_{k}\right) \mathbf{e}_{k}$ is in duality with $\left|\mathbf{e}_{k}\right|^{3} \mathbf{e}_{k}$, and we can repeat the argument to obtain $\mathrm{e}_{k} \in L^{8}\left(I ; V_{k}\right)$, and after another finite number of boot-strapping steps we get the integrability better than $p$. Thus, from (4.18) modified as described above, we eventually get $\mathrm{e}_{k n} \rightarrow \mathrm{e}_{k}$ in $L^{2}\left(Q ; \mathbb{R}^{3}\right)$.

Using (4.2e), we also get $\varrho_{n}^{\prime}\left(\dot{\mathrm{m}}_{k n}\right) \cdot \dot{\mathrm{m}}_{k n} \rightarrow \varrho\left(\dot{\mathrm{m}}_{k}\right)$ in $L^{2}(Q)$. Thus we can see that all terms on the right-hand side of the heat equation $(4.5 \mathrm{~b})$ converges in $L^{1}(Q)$. Thus the limit passage from $(4.5 \mathrm{~b})$ to $(4.47 \mathrm{c})$ can be accomplished.

Moreover, (4.54) can be used for a general $v$ to pass to the limit from $(4.5 \mathrm{~d})$ to (4.47e) if integrated over time.

Proposition 4.2. (Passage $k \rightarrow \infty)$ A selected subsequence $\left\{\left(\mathrm{m}_{k}, \theta_{k}, \mathrm{~h}_{k}, \mathrm{e}_{k}\right)\right\}_{k \in \mathbb{N}}$ does exist such that it converges weakly* in the topologies indicated by (4.19a)(4.19e) and (4.21). Its limit, denoted by $(\mathrm{m}, \theta, \mathrm{h}, \mathrm{e})$, is a weak solution in accordance to Definition 3.1 satisfying also (3.7).

Sketch of the Proof. The main scenario as far as the Gilbert and the heat equation one concerned is as in Proposition 3.10 of Ref. 21 and we outline only the differences and expansions in successive steps. 
Step 1. Passage to the limit in (4.47a). For $\mathrm{s}$ in the unit ball of $L^{2}\left(Q ; \mathbb{R}^{3}\right)$, we choose $\mathbf{w}=\dot{\mathrm{m}}_{k}-\mathrm{s}$ as a test in (4.47b). This choice gives $\int_{Q} \mathrm{r}_{k} \cdot \mathrm{sd} x \mathrm{~d} t \leq \int_{Q} \alpha \dot{\mathrm{m}}_{k} \cdot \mathbf{s}+$ $\rho(\mathbf{s}) \mathrm{d} x \mathrm{~d} t \leq C\left(1+\left\|\dot{\mathrm{m}}_{k}\right\|_{L^{2}\left(Q ; \mathbb{R}^{3}\right)}^{2}\right)$. Hence by $(4.21 \mathrm{~b}), \mathbf{r}_{k}$ is bounded in $L^{2}\left(Q ; \mathbb{R}^{3}\right)$ and (up to a subsequence) has a weak limit $r \in L^{2}\left(Q ; \mathbb{R}^{3}\right)$. The passage to the limit in the other terms is possible, thanks to the uniform bounds (4.19a), (4.19c), (4.19d) and (4.21a), (4.21b), (4.21d). In fact, by the estimate (4.19a), $\nabla \mathrm{m}_{k}$ converges weakly in $L^{2}\left(Q ; \mathbb{R}^{3 \times 3}\right)$. Furthermore, in view of $(4.21 \mathrm{~b}), \dot{\mathrm{m}}_{k}$ converges weakly to $\dot{\mathrm{m}}$ in $L^{2}\left(Q ; \mathbb{R}^{3}\right)$, whereas $\mathrm{m}_{k}$ converges strongly in $L^{p^{\star}}\left(I ; L^{q^{\star}}\left(\Omega ; \mathbb{R}^{3}\right)\right)$ for all $p^{\star} \in[1, \infty)$ and $q^{\star} \in[1,6)$ by Aubin-Lions' theorem. Thus, by $(3.2 \mathrm{~b}), \varphi_{0}^{\prime}\left(\mathrm{m}_{k}\right)$ converges to $\varphi_{0}^{\prime}(\mathrm{m})$ in $L^{1}\left(Q ; \mathbb{R}^{3}\right)$ and, by $(3.2 \mathrm{c}), g^{-1}\left(\left|\mathrm{~m}_{k}\right|\right) \mathrm{m}_{k} \times \dot{\mathrm{m}}_{k}$ converges to $g^{-1}(|\mathrm{~m}|) \mathrm{m} \times \dot{\mathrm{m}}$ in $L^{1}\left(Q ; \mathbb{R}^{3}\right)$. By $(4.19 \mathrm{c}), \mathrm{h}_{k}$ converges weakly ${ }^{*}$ to a limit $\mathrm{h}$ in $L^{\infty}\left(I ; L^{2}\left(\Omega ; \mathbb{R}^{3}\right)\right)$. By $(4.19 \mathrm{~d}), \theta_{k}$ converges weakly* to a limit $\theta$ in $L^{\infty}\left(I ; L^{\omega}(\Omega)\right)$, respectively. From the weak convergence of $\theta_{k}$, the strong convergence of $\mathrm{m}_{k}$, and the continuity of $\varphi_{1}^{\prime}(\cdot)$ we obtain the convergence of $\theta_{k} \varphi_{1}^{\prime}\left(\mathrm{m}_{k}\right)$ in $L^{1}\left(Q ; \mathbb{R}^{3}\right)$. We thus conclude that the weak limits $\mathrm{r}, \mathrm{m}, \theta$ and h satisfy (3.3a).

Step 2. Passage to the limit in (4.47b). We choose $\mathbf{z}=\dot{\mathrm{m}}_{k}$ as test function in (4.47a). By the convergence results established in Step 1, and by the weak-lower semicontinuity of the $L^{2}$ norm, we obtain

$$
\begin{aligned}
\limsup _{n \rightarrow \infty} & \int_{Q} \mathrm{r}_{k} \cdot \dot{\mathrm{m}}_{k} \mathrm{~d} x \mathrm{~d} t \\
= & \limsup _{k \rightarrow \infty} \int_{Q}\left(\frac{\mathrm{m}_{k} \times \dot{\mathrm{m}}_{k}}{g\left(\left|\mathrm{~m}_{k}\right|\right)}-\varphi_{0}^{\prime}\left(\mathrm{m}_{k}\right)-\theta_{k} \varphi_{1}^{\prime}\left(\mathrm{m}_{k}\right)+\mu_{0} \mathrm{~h}_{k}+\mu_{0} \mathrm{~h}_{\mathrm{e}}\right) \\
& \cdot \dot{\mathrm{m}}_{k}-\lambda \nabla \mathrm{m}_{k}: \nabla \dot{\mathrm{m}}_{k} \mathrm{~d} x \mathrm{~d} t \\
= & \lim _{n \rightarrow \infty} \int_{Q}\left(\mu_{0} \mathrm{~h}_{k}+\mu_{0} \mathrm{~h}_{\mathrm{e}}-\varphi_{0}^{\prime}\left(\mathrm{m}_{k}\right)-\theta_{k} \varphi_{1}^{\prime}\left(\mathrm{m}_{k}\right)\right) \cdot \dot{\mathrm{m}}_{k} \mathrm{~d} x \mathrm{~d} t \\
& -\limsup _{n \rightarrow \infty} \int_{\Omega} \frac{\lambda}{2}\left|\nabla \mathrm{m}_{k}(T, \cdot)\right|^{2} \mathrm{~d} x+\lim _{n \rightarrow \infty} \int_{\Omega} \frac{\lambda}{2}\left|\nabla \mathrm{m}_{k}(0, \cdot)\right|^{2} \mathrm{~d} x \\
\leq & \int_{Q}\left(\mu_{0} \mathrm{~h}+\mu_{0} \mathrm{~h}_{\mathrm{e}}-\varphi_{0}^{\prime}(\mathrm{m})-\theta \varphi_{1}^{\prime}(\mathrm{m})\right) \cdot \dot{\mathrm{m}}_{\mathrm{d} x} \mathrm{~d} t \\
& -\int_{\Omega} \frac{\lambda}{2}|\nabla \mathrm{m}(T, \cdot)|^{2}-\frac{\lambda}{2}|\nabla \mathrm{m}(0, \cdot)|^{2} \mathrm{~d} x .
\end{aligned}
$$

It follows from (3.3a) that $\Delta \mathrm{m} \in L^{2}\left(Q ; \mathbb{R}^{3}\right)$. Moreover, since $\mathrm{m} \in L^{2}\left(I ; W^{1,2}\left(\Omega ; \mathbb{R}^{3}\right)\right) \cap$ $W^{1,2}\left(I ; L^{2}\left(\Omega ; \mathbb{R}^{3}\right)\right)$, the function $[0, T] \ni t \mapsto \nabla \mathrm{m}(t, \cdot) \in L^{2}\left(\Omega ; \mathbb{R}^{3 \times 3}\right)$ is weakly continuous. Thus, the following by-parts integration formula holds:

$$
\frac{1}{2} \int_{\Omega}|\nabla \mathrm{m}(T, \cdot)|^{2}-|\nabla \mathrm{m}(0, \cdot)|^{2} \mathrm{~d} x=\int_{Q} \Delta \mathrm{m} \cdot \dot{\mathrm{m}} \mathrm{d} x \mathrm{~d} t .
$$


Combining (4.56), (4.57) and (3.3a) we obtain

$$
\begin{aligned}
\limsup _{k \rightarrow \infty} & \int_{Q} \mathrm{r}_{k} \cdot \dot{\mathrm{m}}_{k} \mathrm{~d} x \mathrm{~d} t \\
\quad \leq & \int_{Q}\left(\frac{\mathrm{m} \times \dot{\mathrm{m}}}{g(|\mathrm{~m}|)}+\mu_{0} \mathrm{~h}+\mu_{0} \mathrm{~h}_{\mathrm{e}}-\varphi_{0}^{\prime}(\mathrm{m})-\theta \varphi_{1}^{\prime}(\mathrm{m})\right) \cdot \dot{\mathrm{m}}+\lambda \Delta \mathrm{m} \cdot \dot{\mathrm{m}} \mathrm{d} x \mathrm{~d} t \\
\quad= & \int_{Q} \mathrm{r} \cdot \dot{\mathrm{m}} \mathrm{d} x \mathrm{~d} t .
\end{aligned}
$$

Taking the limsup of both sides of (4.47b) and using (4.58), we arrive at (3.3b).

Step 3. Passage to the limit in (4.47d)-(4.47e). We pass to the limit in the Maxwell equations, i.e. from $(4.47 \mathrm{~d})$ and $(4.47 \mathrm{e})$ to $(3.5)-(3.6)$. This step is also straightforward since the only nonlinearities in these equations involve $\theta_{k}$, for which we have compactness by Aubin-Lions' theorem. Having (3.5) and (3.6) at our disposal, we can extract the important information that

$$
\mathrm{h} \in L^{2}\left(I ; L_{\text {curl }, 0}^{2}\left(\Omega ; \mathbb{R}^{3}\right)\right) \quad \text { and } \quad \dot{\mathrm{h}} \in L^{2}\left(I ; L_{\text {curl }, 0}^{2}\left(\Omega ; \mathbb{R}^{3}\right)^{*}\right)
$$

because, in the sense of distributions on $Q$, we have curlh $=\sigma(\theta) \mathrm{e} \in L^{2}\left(Q ; \mathbb{R}^{3}\right)$ and $\dot{\mathrm{h}}=-(\operatorname{curl} \mathrm{e}+\dot{\mathrm{m}}) / \mu_{0}$ with $\mathrm{e} \in L^{2}\left(Q ; \mathbb{R}^{3}\right)$ and $\dot{\mathrm{m}} \in L^{2}\left(Q ; \mathbb{R}^{3}\right)$.

Step 4. Strong convergence of $\mathrm{e}_{k}$ and $\dot{\mathrm{m}}_{k}$ in $L^{2}\left(Q ; \mathbb{R}^{3}\right)$. This result is preliminarily needed to pass to the limit in the heat equation, a step that we will complete later. To prove that $\mathrm{e}_{k} \rightarrow \mathrm{e}$ in $L^{2}\left(Q ; \mathbb{R}^{3}\right)$ we test the difference of the Galerkin Maxwell equations (4.47d) and (4.47e) and the limit Maxwell equations (3.5) and (3.6) respectively by the difference $\mathrm{e}_{k}-\mathrm{e}$ and $\mathrm{h}_{k}-\mathrm{h}$, similarly how we did it in (4.18). However, to use the Galerkin identity $(4.47 \mathrm{~d})$ and $(4.47 \mathrm{e})$ we should now rather approximate $\mathrm{e}$ and $\mathrm{h}$ into the finite-dimensional subspaces $V_{k}$ and $U_{k}$ by a pair of sequences $\mathrm{e}_{k}^{\text {app }}$ and $\mathrm{h}_{k}^{\text {app }}$ which converge strongly to $\mathrm{e}$ and $\mathrm{h}$, respectively, so that

$$
\begin{array}{cl}
\dot{\mathrm{h}}_{k}^{\text {app }}+\dot{\mathrm{m}}+\operatorname{curl} \mathrm{e}_{k}^{\text {app }}=\mathbf{r}_{k}^{(1)} \rightarrow 0 & \text { in } L^{2}\left(I ; L_{\text {curl, } 0}^{2}\left(\Omega ; \mathbb{R}^{3}\right)^{*}\right), \\
-\operatorname{curlh}_{k}^{\text {app }}+\sigma(\theta) \mathbf{e}_{k}^{\text {app }}=\mathbf{r}_{k}^{(2)} \rightarrow 0 & \text { in } L^{2}\left(Q ; \mathbb{R}^{3}\right),
\end{array}
$$

where we used (4.59). Then we test the difference of the approximated Maxwell equations (4.47d) and (4.47e) and the approximated Maxwell equations (4.60) respectively by the difference $\mathrm{e}_{k}^{\text {app }}-\mathrm{e}_{k}$ and $\mathrm{h}_{k}^{\text {app }}-\mathrm{h}_{k}$. Taking benefit from smoothness of the approximation $\mathrm{e}_{k}^{\text {app }}$ and $\mathrm{h}_{k}^{\text {app }}$, we still have the curl-cancellation $\int_{\mathbb{R}^{3}} \operatorname{curl}\left(\mathrm{e}_{k}-\right.$ $\left.\mathrm{e}_{k}^{\text {app }}\right) \cdot\left(\mathrm{h}_{k}-\mathrm{h}_{k}^{\text {app }}\right)-\operatorname{curl}\left(\mathrm{h}_{k}-\mathrm{h}_{k}^{\text {app }}\right) \cdot\left(\mathrm{e}_{k}-\mathrm{e}_{k}^{\text {app }}\right) \mathrm{d} x=0$. In this way, like in (4.18) after integration over $[0, T]$, we obtain

$$
\begin{aligned}
& \int_{Q} \sigma\left(\theta_{k}\right)\left|\mathrm{e}_{k}^{\mathrm{app}}-\mathrm{e}_{k}\right|^{2} \mathrm{~d} x \mathrm{~d} t \\
& \quad \leq \int_{Q}\left(\sigma\left(\theta_{k}\right)-\sigma(\theta)\right) \mathrm{e}_{k}^{\mathrm{app}} \cdot\left(\mathrm{e}_{k}^{\mathrm{app}}-\mathrm{e}_{k}\right)-\left(\dot{\mathrm{m}}_{k}-\dot{\mathrm{m}}\right) \cdot\left(\mathrm{h}_{k}^{\mathrm{app}}-\mathrm{h}_{k}\right)
\end{aligned}
$$




$$
\begin{aligned}
& +\mathbf{r}_{k}^{(1)} \cdot\left(\mathbf{h}_{k}^{\text {app }}-\mathbf{h}_{k}\right)+\mathbf{r}_{k}^{(2)} \cdot\left(\mathbf{e}_{k}^{\text {app }}-\mathrm{e}_{k}\right) \mathrm{d} x \mathrm{~d} t \\
& +\int_{\Omega} \frac{\mu_{0}}{2}\left|\mathbf{h}_{k}^{\text {app }}(0, \cdot)-\mathrm{h}_{k}(0, \cdot)\right|^{2} \mathrm{~d} x .
\end{aligned}
$$

Let us emphasize that these arguments do not rely on that $\mathrm{h}_{k}^{\text {app }}(T, \cdot)-\mathrm{h}_{k}(T, \cdot)$ would have a good sense in the limit and on the curl-cancellation property in the limit. By Aubin-Lions' theorem, $\theta_{k} \rightarrow \theta$ in $L^{1}(Q)$ and thus $\left(\sigma\left(\theta_{k}\right)-\sigma(\theta)\right) \mathbf{e}_{k}^{\text {app }} \rightarrow 0$ in $L^{2}\left(Q ; \mathbb{R}^{3}\right)$, and by using that $\mathrm{e}_{k}^{\text {app }}-\mathrm{e}_{k}$ is certainly bounded in $L^{2}\left(Q ; \mathbb{R}^{3}\right)$, we have $\left(\sigma\left(\theta_{k}\right)-\sigma(\theta)\right) \mathrm{e}_{k}^{\text {app }} \cdot\left(\mathrm{e}_{k}^{\text {app }}-\mathrm{e}_{k}\right) \rightarrow 0$ in $L^{1}(Q)$. As we already proved $\dot{\mathrm{m}}_{k}-\dot{\mathrm{m}} \rightarrow 0$ in $L^{2}\left(Q ; \mathbb{R}^{3}\right)$ and as $\mathrm{h}_{k}^{\text {app }}-\mathrm{h}_{k}$ is certainly bounded in $L^{2}\left(Q ; \mathbb{R}^{3}\right)$, we have $\left(\dot{\mathrm{m}}_{k}-\dot{\mathrm{m}}\right)$. $\left(\mathrm{h}_{k}^{\text {app }}-\mathbf{h}_{k}\right) \rightarrow 0$ in $L^{1}(Q)$. Now an important point is that the residuum $\mathbf{r}_{k}^{(1)} \in$ $L^{2}\left(I ; L_{\text {curl, } 0}^{2}\left(\Omega ; \mathbb{R}^{3}\right)^{*}\right)$ is in duality with $\mathrm{h}_{k}^{\text {app }}-\mathrm{h}_{k} \in L^{2}\left(I ; L_{\text {curl, } 0}^{2}\left(\Omega ; \mathbb{R}^{3}\right)\right)$ so that the term $\mathbf{r}_{k}^{(1)} \cdot\left(\mathrm{h}_{k}^{\text {app }}-\mathrm{h}_{k}\right)$ converges to zero in $L^{1}(Q)$. Similarly, the residuum $\mathbf{r}_{k}^{(2)} \in$ $L^{2}\left(Q ; \mathbb{R}^{3}\right)$ is in duality with $\mathrm{e}_{k}^{\text {app }}-\mathrm{e}_{k} \in L^{2}\left(\Omega ; \mathbb{R}^{3}\right)$ so that the term $\mathbf{r}_{k}^{(2)} \cdot\left(\mathbf{e}_{k}^{\text {app }}-\mathrm{e}_{k}\right)$ converges to zero in $L^{1}(Q)$. Here, in both of these last terms, we essentially benefit from the eddy-current approximation. Thus, (4.61) gives $\mathrm{e}_{k}^{\text {app }}-\mathrm{e}_{k} \rightarrow 0$ in $L^{2}(Q)$ (here we used $\sigma(\cdot) \geq \sigma_{\min }>0$, cf. (3.2f)) and, since $\mathrm{e}_{k}^{\text {app }} \rightarrow \mathrm{e}$ in $L^{2}(Q)$, we eventually obtain the desired convergence $\mathrm{e}_{k} \rightarrow \mathrm{e}$ in $L^{2}(Q)$.

We omit the proof of the strong convergence of $\dot{\mathrm{m}}_{k}$, which can be performed in the same manner as in Eqs. (3.68) and (3.69) of Ref. 21 (using very subtle arguments, in particular $\nabla \mathrm{m} \in L^{2}\left(Q ; \mathbb{R}^{3 \times 3}\right)$ which needs (3.2c) etc.) modified here by adding the inequality $\int_{Q} \varrho(\dot{\mathrm{m}}) \mathrm{d} x \mathrm{~d} t \leq \liminf _{k \rightarrow \infty} \int_{Q} \varrho\left(\dot{\mathrm{m}}_{k}\right) \mathrm{d} x \mathrm{~d} t$.

Step 5. Passage to the limit in (4.47c). By (4.19d), (4.21a) and (4.21d), we have weak* convergence of $\theta_{k}$ in $L^{\infty}\left(I ; L^{\omega}(Q)\right)$ and weak convergence of $\nabla \theta_{k}$ in $L^{r}\left(Q ; \mathbb{R}^{3}\right)$. Then, by Aubin-Lions' theorem, $\theta_{k}$ converges strongly in $L^{1+2 \omega / 3}(Q)$. The strong convergence of $\theta_{k}$ along with the weak convergence of its gradient allow us to pass to the limit on the left-hand side of $(4.47 \mathrm{c})$. We then consider the right-hand side of $(4.47 \mathrm{c})$. By Step 4, the term $\sigma\left(\theta_{k}\right)\left|\mathrm{e}_{k}\right|^{2}+\alpha\left|\dot{\mathrm{m}}_{k}\right|^{2}$ converges strongly to $\sigma(\theta)|\mathrm{e}|^{2}+\alpha|\dot{\mathrm{m}}|^{2}$ in $L^{1}(Q)$; by the continuity and the degree- 1 homogeneity of $\rho$, the strong convergence of $\dot{\mathrm{m}}_{k}$ guarantees that $\rho\left(\dot{\mathbf{m}}_{k}\right)$ converges strongly to $\rho(\dot{\mathbf{m}})$. The passage to the limit in the term $\theta_{k} \varphi\left(\mathrm{m}_{k}\right) \cdot \dot{\mathrm{m}}_{k}$ follows from the strong convergence of $\theta_{k}, \mathrm{~m}_{k}$ and $\dot{\mathrm{m}}_{k}, L^{2}\left(\Omega ; \mathbb{R}^{3}\right)$. The remaining terms in the last line of $(4.47 \mathrm{c})$ converge because of $(4.3)$.

Remark 4.1. (Positivity of temperature) If (3.2j) and (3.2m) are strengthened by requiring $\varphi_{1}^{\prime}$ bounded and $\inf \theta_{0}>0$, one can also strengthen Lemma 4.2 to obtain even positivity of $\theta$ by a comparison argument as devised in Sec. 4.2.1 of Ref. 12. Knowing already $\theta_{k n} \geq 0$ from Lemma 4.2, we deduce from (4.5b) that

$$
\begin{aligned}
c\left(\theta_{k n}\right) \dot{\theta}_{k n}-\operatorname{div}\left(\kappa\left(\theta_{k n}\right) \nabla \theta_{k n}\right) & \geq \alpha\left|\dot{\mathrm{m}}_{k n}\right|^{2}+\theta_{k n} \varphi_{1}^{\prime}\left(\mathrm{m}_{k n}\right) \cdot \dot{\mathrm{m}}_{k n} \\
& \geq-\frac{1}{4 \alpha}\left|\theta_{k n} \varphi_{1}^{\prime}\left(\mathrm{m}_{k n}\right)\right|^{2} \\
& \geq-\frac{\sup \varphi_{1}^{\prime}(\cdot)}{4 \alpha}\left|\theta_{k n}\right|^{2} .
\end{aligned}
$$


We compare the solution to (4.5b) with the solution to the ordinary-differential equation $c(\chi) \dot{\chi}+\frac{\sup \varphi_{1}^{\prime}(\cdot)}{4 \alpha}|\chi|^{2}=0$ which, for $\chi(0)=\inf \theta_{0}>0$, gives a sub-solution of the heat equation $(4.5 \mathrm{~b})$, exploiting also the fact that $\theta_{\mathrm{e}, k} \geq 0$ a.e. in $\partial \Omega$. Taking also into account (3.2d), we have

$$
\frac{\sup \varphi_{1}^{\prime}(\cdot)|\chi|^{2}}{4 \alpha c(\chi)} \leq \frac{\sup \varphi_{1}^{\prime}(\cdot)|\chi|^{2}}{4 \alpha c_{\min }\left(1+|\chi|^{\omega-1}\right)}
$$

and thus we can still estimate $\theta_{k n}(t, x) \geq \chi(t)$ if $\chi$ solves the initial-value problem

$$
\dot{\chi}+\frac{\sup \varphi_{1}^{\prime}(\cdot)|\chi|^{2}}{4 \alpha c_{\min }\left(1+|\chi|^{\omega-1}\right)}=0, \quad \chi(0)=\inf \theta_{0}>0 .
$$

This problem possesses a unique solution which is decreasing in time but ever positive; here it is important that the nonlinearity $\xi \mapsto|\chi|^{2} /\left(1+|\chi|^{\omega-1}\right)$ is Lipschitz locally continuous. Thus $\theta_{k n} \geq \min _{[0, T]} \chi(\cdot)>0$. This positivity is "uniform" and is thus preserved under the limit for $k \rightarrow \infty$ and $n \rightarrow \infty$.

\section{Concluding Remarks}

In this last section we briefly comment some possible generalizations and modifications, and also those generalizations that seem to bring serious difficulties.

Remark 5.1. (Temperature dependent coefficients) One can consider temperaturedependent coefficients $\alpha=\alpha(\theta), \rho=\rho(\theta)$ in (2.15), and $g=g(m, \theta)$ in addition to already considered temperature-dependence of $\kappa$ and $\sigma$. Under a suitable qualification, this generalization is relatively routine because there is a compactness by Aubin-Lions' theorem in $\theta$.

Remark 5.2. (Electromagnetic field outside) Considering the full Maxwell system, i.e. with $\varepsilon_{0} \dot{\mathrm{e}}$ added in $(2.1 \mathrm{~d})$, would make the whole problem hyperbolic/parabolic and bring substantial difficulties. For example, there would not be any estimate on curlh in $L^{2}\left(Q ; \mathbb{R}^{3}\right)$ and the argument used to limit (4.61) would fail. This is also why we confined ourselves only on the magnet itself where the eddy-current approximation of the Maxwell system is well acceptable and why we neglected the electromagnetic field outside which otherwise would standardly be considered as the full Maxwell system, cf. Sec. 5 of Ref. 29.

Remark 5.3. (Thermoelectrical effects) Interesting generalization would be to consider Thomson's thermoelectrical effect, see e.g. Sec. 3.2.2 of Ref. 20. Instead of (2.6), one then considers

$$
\begin{aligned}
& \mathbf{q}=-\kappa(\theta) \nabla \theta+\beta_{\mathrm{S}}(\theta) \mathrm{e} \\
& \mathrm{J}=\sigma(\theta) \mathrm{e}+\beta_{\mathrm{P}}(\theta) \nabla \theta
\end{aligned}
$$


The coefficient $\beta_{\mathrm{S}}$ is related to Seebeck's cross effect and $\beta_{\mathrm{P}}$ is responsible for Peltier's effects. It is physically well justified to impose the following (Nobel-prize awarded) Onsager symmetry condition (see also Ref. 17):

$$
\beta_{\mathrm{S}}(\theta)=\theta \beta_{\mathrm{P}}(\theta) \text {. }
$$

The system (2.1) would then become

$$
\begin{aligned}
& \frac{1}{g(|\mathrm{~m}|)} \mathrm{m} \times \dot{\mathrm{m}}+\lambda \Delta \mathrm{m}-\varphi_{0}^{\prime}(\mathrm{m})-\theta \varphi_{1}^{\prime}(\mathrm{m})+\mu_{0}\left(\mathrm{~h}+\mathrm{h}_{\mathrm{e}}\right)-\alpha \dot{\mathrm{m}} \in \partial \varrho(\dot{\mathrm{m}}), \\
& c(\theta) \dot{\theta}-\operatorname{div}\left(\kappa(\theta) \nabla \theta-\beta_{\mathrm{S}}(\theta) \mathrm{e}\right) \\
& =\sigma(\theta)|\mathrm{e}|^{2}+\beta_{\mathrm{P}}(\theta) \nabla \theta \cdot \mathrm{e}+\alpha|\dot{\mathrm{m}}|^{2}+\varrho(\dot{\mathrm{m}})+\theta \varphi_{1}^{\prime}(\mathrm{m}) \cdot \dot{\mathrm{m}}, \\
& \mu_{0}(\dot{\mathrm{h}}+\dot{\mathrm{m}})+\operatorname{curl} \mathrm{e}=0, \\
& \varepsilon_{0} \dot{\mathrm{e}}-\operatorname{curlh}+\sigma(\theta) \mathrm{e}+\beta_{\mathrm{P}}(\theta) \nabla \theta=0,
\end{aligned}
$$

with boundary conditions (2.2). Thanks to (5.2), the system (5.3) enjoys formally a lot of various cancellation properties: in addition to curl-cancellation in the Maxwell system (5.3c) and (5.3d) under the test by $\mathrm{h}$ and $\mathrm{e}$ and the cancellation of the dissipative and adiabatic terms under the test of the whole system (5.3) by $\mathrm{m}, 1, \mathrm{~h}$ and $\mathrm{e}$ as before, also the cross-coupling terms in the heat equation (5.3b) itself mutually cancel when tested by $1 / \theta$, and still we have the cancellation of the Joule heat and Peltier's terms when tested $(5.3 \mathrm{~b})-(5.3 \mathrm{~d})$ by $\theta, \mathrm{h}$ and $\mathrm{e}$.

Under suitable qualification of $\beta_{\mathrm{S}}$ and $\beta_{\mathrm{P}}$, a lot of the above considerations seem possible to be expanded for these cross-terms, too. However, e.g. the strategy (4.61) would augment by the term $\left(\beta_{\mathrm{P}}\left(\theta_{k}\right) \nabla \theta_{k}-\beta_{\mathrm{P}}(\theta) \nabla \theta\right) \cdot\left(\mathrm{e}_{k}^{\text {app }}-\mathrm{e}_{k}\right)$ which does not seem possible to push to zero for $k \rightarrow \infty$.

\section{Acknowledgments}

The partial support of this research from the grants 106/09/1573 and 201/09/0917 (GA ČR), A 100750802 (GA AV ČR), LC 06052 and MSM 21620839 (MŠMT ČR), as well as the research plan AV0Z20760514 ( $\breve{\mathrm{CR}})$ is warmly acknowledged. G. T. acknowledges support from GNFM's Progetto Giovani 2009 — "Modellazione fisico-matematica di materiali e strutture intelligenti".

\section{References}

1. A. Alonso, A mathematical justification of the low-frequency heterogeneous timeharmonic Maxwell equations, Math. Models Methods Appl. Sci. 9 (1999) 475-489.

2. F. Alouges and A. Soyeur, On global weak solutions for Landau-Lifshitz equations: Existence and nonuniqueness, Nonlinear Anal. 18 (1992) 1071-1084.

-3. H. Ammari, A. Buffa and J.-C. Nédélec, A justification of eddy currents model for the Maxwell equations, SIAM J. Appl. Math. 60 (2000) 1805-1823.

-4. W. Baltensperger and J. Helman, Dry friction in micromagnetics, IEEE Trans. Mag. 27 (1991) 4772-4774. 
5. G. Bertotti, Hysteresis in Magnetism (Academic Press, 1998).

6. M. Bertsch, P. Podio Guidugli and V. Valente, On the dynamics of deformable ferromagnets. I. Global weak solutions for soft ferromagnets at rest, Ann. Mat. Pura Appl. 179 (2001) 331-360.

7. W. Brown, Micromagnetics (Wiley, 1963).

8. G. Carbou and P. Fabrie, Regular solutions for Landau-Lifshitz equation in $\mathbb{R}^{3}$, Commun. Appl. Anal. 5 (2001) 17-30.

9. Y. Chen and B. Guo, Two-dimensional Landau-Lifshitz equation, J. Partial Diff. Eqns. 9 (1996) 313-322.

10. S. Chikazumi, Physics of Ferromagnetism (Oxford Univ. Press, 1997).

11. G. Duvaut and J.-L. Lions, Inequalities in Mechanics and Physics (Springer-Verlag, 1976).

12. E. Feireisl, H. Petzeltová and E. Rocca, Existence of solutions to a phase transition model with microscopic movements, Math. Methods Appl. Sci. 32 (2009) 1345-1369.

13. M. Fabrizio and A. Morro, Electromagnetism of Continuous Media: Mathematical Modelling and Applications (Oxford Univ. Press, 2004).

14. T. Gilbert, A Lagrangian formulation of the gyromagnetic equation of the magnetization field, Phys. Rev. 100 (1955) 1243.

15. V. Girault and P.-A. Raviart, Finite Element Approximation of the Navier-Stokes Equations (Springer-Verlag, 1979).

16. B. Guo and S. Ding, Landau-Lifshitz Equations (World Scientific, 2008).

17. L. Landau, E. Lifshitz and L. Pitaevskii, Electrodynamics of Continuous Media (Pergamon, 1984).

18. L. Landau and E. Lifshitz, On the theory of dispersion of magnetic permeability inferromagnetic bodies, Phys. Z. Sowjet. 8 (1935) 153-169.

19. C. Melcher, Existence of partially regular solutions for Landau-Lifshitz equations in $\mathbb{R}^{3}$, Comm. Partial Diff. Eqns. 30 (2005) 567-587.

20. H. C. Öttinger, Beyond Equilibrium Thermodynamics (Wiley, 2005).

21. P. Podio-Guidugli, T. Roubíček and G. Tomassetti, A thermodynamically-consistent theory of the ferro/paramagnetic transition, Preprint No. 683, Universita degli Studi di Roma II "Tor Vergata", Arch. Rational Mech. Anal., to appear.

22. P. Podio-Guidugli and G. Tomassetti, Magnetization switching with nonstandard dissipation, IEEE Trans. Mag. 42 (2006) 3652-3656.

23. K. R. Rajagopal and T. Roubíček, On the effect of dissipation in shape-memory alloys, Nonlinear Anal.: Real World Appl. 4 (2003) 581-597.

24. T. Roubíček, Nonlinear Partial Differential Equations with Applications (Birkhäuser, 2005).

25. T. Roubíček and G. Tomassetti, Thermodynamics of shape-memory alloys under electric current, Z. Angew. Math. Phys. 61 (2010) 1-20.

26. T. Roubíček, G. Tomassetti and C. Zanini, The Gilbert equation with dry-friction-type damping, J. Math. Anal. Appl. 355 (2009) 453-468.

27. A. Visintin, On Landau-Lifshitz equations for ferromagnetism, Jpn. J. Appl. Math. 2 (1985) 69-84.

28. A. Visintin, Modified Landau-Lifshitz equation for ferromagnetism, Physica B 233 (1997) 365-369.

29. A. Visintin, Maxwell's equations with vector hysteresis, Arch. Rational Mech. Anal. 175 (2005) 1-37. 
This article has been cited by:

1.N.BELLOMO nicola.bellomo@polito.it J. MÁLEK malek@karlin.mff.cuni.cz. 2011. CONTINUUM THERMODYNAMICS AND NONLINEAR PARTIAL DIFFERENTIAL EQUATIONS. Mathematical Models and Methods in Applied Sciences 21:01, 1-6. [Citation] [References] [PDF] [PDF Plus] 